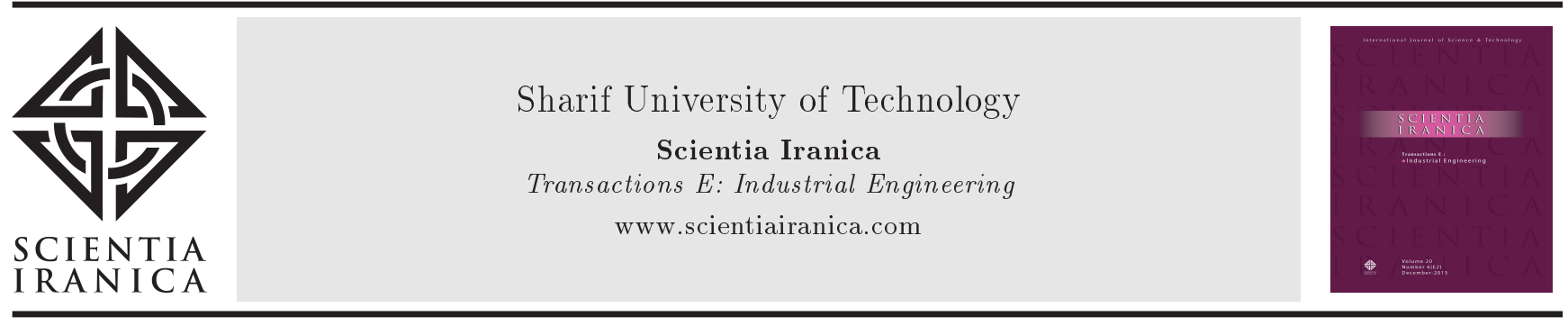

\title{
A prototype-based classification using extended imperialist competitive algorithm
}

\author{
M.H. Fazel Zarandi ${ }^{a, *}$, M. Teimouri ${ }^{\mathrm{b}}$, A. Zaretalab ${ }^{\mathrm{a}}$ and V. Hajipour ${ }^{\mathrm{c}}$ \\ a. Department of Industrial Engineering, Amirkabir University of Technology, 424 Hafez Avenue, Tehran, Iran. \\ b. Faculty of Industrial and Mechanical Engineering, Qazvin Branch, Islamic Azad University, Qazvin, Iran. \\ c. Department of Industrial Engineering, Faculty of Engineering, Bu-Ali Sina University, Hamedan, Iran.
}

Received 4 July 2015; received in revised form 26 December 2015; accepted 26 December 2016

\author{
KEYWORDS \\ Prototype-based \\ classification; \\ Imperialist \\ competitive \\ algorithm; \\ UCI machine learning \\ repository.
}

\begin{abstract}
Classification is an important machine learning technique used to predict group membership for data instances. In this paper, we propose an efficient prototypebased classification approach in the data classification literature by a novel soft-computing approach based on extended imperialist competitive algorithm. The novel classifier is called EICA. The goal is to determine the best places of the prototypes. EICA is evaluated under three different fitness functions on twelve typical test datasets from the UCI Machine Learning Repository. The performance of the proposed EICA is compared with well-developed algorithms in classification including original Imperialist Competitive Algorithm (ICA), the Artificial Bee Colony (ABC), the Firefly Algorithm (FA), the Particle Swarm Optimization (PSO), the Gravitational Search Algorithm (GSA), the Grouping Gravitational Search Algorithm (GGSA), and nine well-known classification techniques in the literature. The analysis results show that EICA provides encouraging results in contrast to other algorithms and classification techniques.
\end{abstract}

(C) 2017 Sharif University of Technology. All rights reserved.

\section{Introduction}

Machine Learning (ML), as a branch of artificial intelligence, attempts to improve systems' performance [1-3]. ML's classification is the problem of identifying which set of categories (subpopulations) a new observation belongs to, on the basis of a training set of data containing observations (or instances) whose category membership is known. Classification techniques have been extensively applied to several fields such as business, image recognition, biology, medicine, and microarray data analysis, among others. For more details about classification problem, one can refer to $[4,5]$.

The classification is also considered as two distinct

*. Corresponding author. Tel.: 021 64545378

E-mail addresses: zarandi@aut.ac.ir (M.H. Fazel Zarandi); m66.teimouri@gmail.com (M. Teimouri); arash_zaretalab@yahoo.com (A.ZZaretalab); v.hajipour@basu.ac.ir (V.Hajipour) meanings: unsupervised learning (or clustering) and supervised learning. Clustering is a classification process to put data into specific groups or clusters based on their similarities. As a simple explanation, the clustering algorithms can be classified into two categories: hierarchical clustering and partitional clustering [6]. Hierarchical clustering techniques group data objects with a sequence of partitions, either from singleton clusters towards a cluster based on all individuals, or vice versa [7]. Unlike the hierarchical clustering algorithms, the goal of partitional algorithms is to classify the clusters at once based on a criterion function [7]. The most well-known class of partitional clustering methods is the center-based clustering algorithms. The aim of the center-based algorithms is to minimize its objective function. K-means is one of the best-developed center-based clustering algorithms [8]. This paper concentrates on supervised learning as a classification concept. 
Over the past half century, many classification techniques have been presented such as Artificial Neural Networks (ANNs) [9,10], Support Vector Machine (SVM) [11,12], Decision Tree (DT) [13,14], and Instance Based (IB) [15-18]. Among these techniques, the DT technique is simple and easy to use and comprehend by human beings. The SVM technique is a new machine learning method based on the foundation of statistical learning theory introduced by Vapnik [19]. SVM is based on the hypothesis in which the training samples have a certain distribution, restricting its application scope. The ANNs techniques, inspired by biological neural networks [4], attempt to formulate data as a mathematical model for both quantitative and qualitative data. The advantage of this method is the ability to deal with noisy data.

Among these, the IB classifier is one of the most traditional types of classifiers in which the main distinctive characteristic is to apply the instances themselves as class representations. The IB classification relies on the closeness or similarity between instances selected as a representative (prototype) of the learned class and the new examples to be classified. If the representative instances were previously observed exemplars, we would apply classifiers called memory-based, case-based, or exemplar-based. If the representative instances were abstracted from the given data, we would classify them as prototype-based [16]. The main advantage of the prototype-based classifiers is offering an intuitive summation of the given data in a few number of prototypes, leading, therefore, to plausible and interpretable class structures and classification schemes [20].

Some recent developments show that some hybrid IB classifiers, such as the Prototype Exemplar Learning Classifier (PEL-C) [16] and the Total Recognition by Adaptive Classification Experiments (T.R.A.C.E.) [21], which generalize both prototype-based and exemplarbased classifiers, can be used to discover the "typicality structure" of the learnt category, detecting how the class is decomposable in some subclasses and their typicality grade within the class [22].

There are lots of prototype-based algorithms which are different in terms of the number of the used prototypes and their selection method such as K-Means Classifier (KMC) [4], Learning Vector Quantization (LVQ) [23], Nearest Neighbor Classifier (NNC) [24], Nearest Mean Classifier (NMC) [4], Nearest Subclass Classifier (NSC) [25], and so forth [26,27]. Briefly, NNC does not abstract the data, but rather applies all the training data to label unseen patterns. NMC saves one prototype for each class (i.e., mean of each class), while KMC and LVQ select a fixed number of prototypes per class as an over-fitting avoidance strategy. In LVQ, the training points attract prototypes of the correct class and repel other prototypes. In the learning phase, the degree of attraction and repellence is controlled, which is tended towards zero at each iteration. NSC attempts to impose the number of prototypes per class by introducing a variance constraint parameter. In NSC, every pattern contains the same amount of noise and does not formulate the label noise [17]. However, such assumptions may not be satisfied in real world conditions.

Determining a proper classifier for a classification problem is still a complicated issue. However, various factors are effective in choosing a suitable classifier, including the size of the dataset, the type of hypothesized space representations, having either noisy or noise-free data, and so forth [2]. In the literature, many researchers have used soft-computing approaches for prototype-based techniques. The main reason for that comes from the nature of ML techniques. Since the training process is overly effective in the performance of the algorithms, inappropriate training can be converted to a local optimum, and consequently, increase the number of misclassifications [28]. De Falco et al. [29] introduced an approach based on Particle Swarm Optimization (PSO) for classification. They applied three different fitness functions as minimization to evaluate the quality of solutions. Karaboga and Ozturk [30] presented an Artificial Bee Colony (ABC) algorithm for classification problem inspired by the foraging behavior of honey-bees. They have utilized only one of the fitness functions presented by De Falco et al. [29]. Senthilnath et al. [31] proposed an algorithm based on the Firefly Algorithm (FA) for solving classification problems and tested its proficiency on real datasets. Similar to [30], they also applied one of the functions proposed by De Falco et al. [29]. Bahrololoum et al. [28] proposed a Gravitational Search Algorithm (GSA) to face the classification of instances in multi-class datasets. Recently, Dowlatshahi and Nezamabadi-pour [32] have presented a Grouping Gravitational Search Algorithm (GGSA) for data clustering. There are two significant advantages of GGSA algorithm. First, a special encoding scheme, called grouping encoding, is used in order to make the relevant structures of clustering problems become parts of solutions. Second, given the encoding, special GSA updating equations suitable for the solutions with grouping encoding are used.

Since optimization algorithms are generalpurpose techniques, the results obtained by the aforementioned algorithms confirm that optimization algorithms have high capability for classification problems. Besides, there is no classifier that works best in classification problems. The Imperialist Competitive Algorithm (ICA), introduced by Atashpaz and Lucas [33] and Atashpaz et al. [34], is an evolutionary algorithm based on human's socio-political evolution. This evolutionary optimization algorithm has 
an appropriate performance in many optimization problems such as inventory control [35], data clustering [36], scheduling [37], reliability [38], game theory [39], supply chain [40], project scheduling [41], dynamic facility layout [42], Euclidean minimal spanning tree [43], global optimization [44], and so on. Recently, Hosseini and Al Khaled [45] have reviewed the underlying ideas of how ICA and its application to the engineering disciplines mainly in industrial engineering have emerged. Not only is ICA easy to implement and computationally efficient, but it also has few parameters. However, it is still in its infancy and undergoing intensive studies to improve its performance.

To enhance the quality of the solution in the ICA, several approaches have been proposed in the literature. Zhang et al. [46] proposed an improved algorithm based on the concept of small probability perturbation. They showed that there is no restriction on the number of optimized variables. Razzaghpour and Rusu [47] proposed a modified ICA to multidimensional problems. Their algorithm is compared with the original ICA and Genetic Algorithm (GA) on the circuit-related case study. Duan et al. [48] presented a chaotic ICA. They showed that the chaos can improve the global convergence of ICA and avoid getting stuck at local minima. Kaveh and Talatahari [49] proposed an improved ICA in which the movement of the colonies toward their related imperialist is modified. Also, this approach is applied to our ICA for classification problems. Recently, Al Khaled and Hosseini [44] have proposed a Fuzzy Adaptive Imperialist Competitive Algorithm (FAICA) for global optimization in which the deviation angle parameter is adaptively adjusted using a fuzzy controller. By implementing fuzzy controller, the algorithm was capable of making a balance between global search and local search. Our proposed ICA determines a better quality solution with modifying two procedures: (I) assimilation policy and (II) revolution strategy. Considering these changes, we present an Extended Imperialist Competitive Algorithm (EICA).

In this paper, two phases are considered for the design prototype classifier based on the EICA. In the first phase, the fundamental problem is to determine favorable prototypes for each class which has significant effect on the final results. Therefore, to find these prototypes, EICA is run until the termination criteria are satisfied. The prototypes are obtained by selecting $75 \%$ of the given dataset which is called as a training set. In the second phase, according to the result obtained in the previous phase, each instance from the remaining $25 \%$ of dataset, called the test set, is assigned to the prototype which has the minimum distance. In this respect, Euclidian distance, which is the most well-known and applicable distance, is applied. In order to evaluate the performance of the proposed algorithm, 12 distinguished and standard datasets are chosen from the UCI machine learning repository [50]. The results of the proposed EICA are compared with those of the 15 well-known classification techniques reported in the literature. The fitness functions in our EICA algorithm are exactly the same as the fitness function suggested by De Falco et al. [29]. The performance of the algorithms is measured by Classification Error Percentage (CEP). We attempt to illustrate the performance of the proposed EICA for classification of instances.

The remainder of the paper is organized as follows: In Section 2, ICA is presented. In Section 3, EICA is illustrated in detail. In Section 4, classification using EICA is discussed. Experimental implementation is introduced in Section 5. Then, in Section 6, feasibility of EICA is demonstrated and compared with the results of other classifiers for different datasets and the behavior of optimization algorithm is investigated, and so more discussion is provided. At the end, conclusions and remarks for future research studies are given.

\section{Imperialist Competitive Algorithm (ICA)}

ICA is a new population-based stochastic search algorithm inspired by imperialistic competition introduced by Atashpaz and Lucas [33] for optimization problems. Formerly, ICA has been victorious in solving optimization problems such as design of plate-fin heat exchangers [44], design of a linear induction motor [45], mixed-model assembly line sequencing problem [46], and skin color segmentation [47].

The main framework of ICA consists of 8 steps: generation of the initial empire; assimilation; revolution; exchanging positions of the imperialist and a colony; total power of an empire; imperialistic competition; elimination of the powerless empires; convergence. The ICA's steps are described in detail in the following subsections.

\section{Step 1: Generating initial empires}

Since representing the solutions is the main step in designing an optimization algorithm, an array of decision variables of the problem is considered to encode the problem. For example, in Genetic Algorithm (GA), this format is called "chromosome". In ICA, representation of the solution is called "country". In an $N_{\text {var-dimensional optimization }}$ problem, country is a $1 \times N_{\text {var }}$ array defined as:

$$
\text { Country }=\left[P_{1}, P_{2}, \cdots, P_{N_{\mathrm{var}}}\right] \text {, }
$$

where $P_{i}$ refers the variables of the problem. Figure 1 shows the interpretation of the country using some of socio-political characteristics. The cost of the country is given by assessing cost function $f$ defined by: 


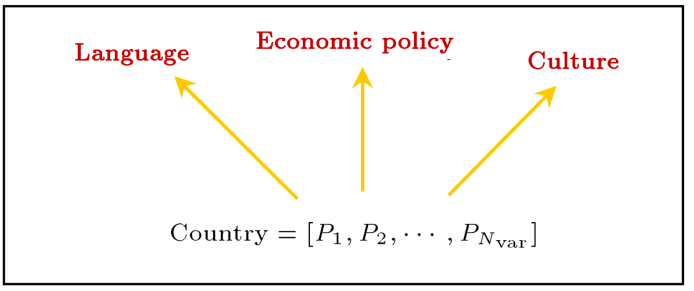

Figure 1. The candidate solutions of the problem, called country.

$$
\text { Cost }=f(\text { Country })=f\left(P_{1}, P_{2}, \cdots, P_{N_{\text {var }}}\right) .
$$

To begin ICA, an initial random population of size $N_{\text {pop }}$ is created, and then, the objective function for all countries is computed. The most powerful countries $\left(N_{\text {imp }}\right)$ are selected as imperialists, and the remaining of the population are colonies $\left(N_{\mathrm{col}}\right)$ that should be assigned to the imperialist. Now, we have two types of countries: imperialist and colony.

The colonies are distributed among the imperialists with respect to their power. To fulfill this purpose, the normalized cost of an imperialist is defined by:

$$
C_{n}=c_{n}-\max _{i}\left\{c_{i}\right\} \quad i=1,2, \cdots, N_{\text {imp }},
$$

where $c_{n}$ and $C_{n}$ are the cost of $n$th imperialist and its normalized cost, respectively. Then, the normalized power of each imperialist is obtained as follows:

$$
P_{n}=\left|\frac{C_{n}}{\sum_{i=1}^{N_{\text {imp }}} C_{i}}\right| .
$$

In Eq. (4), $P_{n}$ is the power of $n$th imperialist. Finally, the initial number of colonies for $n$th empire is computed by:

$$
N C_{n}=\operatorname{round}\left(P_{n} \times N_{\text {col }}\right),
$$

where $N C_{n}$ is the initial number of colonies of $n$th empire, and $N_{\text {col }}$ is the total number of initial colonies. For each imperialist, $N C_{n}$ of the colonies is randomly selected and assigned; then, all together constitute $n$th empire. Thus, if the empire is stronger, its colonies are greater; if the empire is weaker, its colonies are fewer. The initialization process is plotted schematically in Figure 2;

- Step 2: Assimilation

After all the colonies among imperialists are distributed and the initial empires are created, the assimilation policy should be executed. According to this policy, all the colonies are moved and gotten closer to their relevant imperialist based on various socio-political axes (e.g., the culture, language, and economical policy). This movement is depicted in

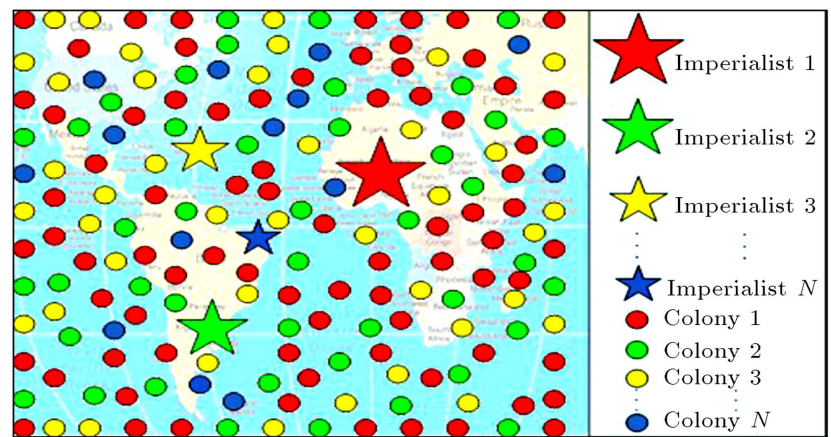

Figure 2. Generating the initial empires [30].

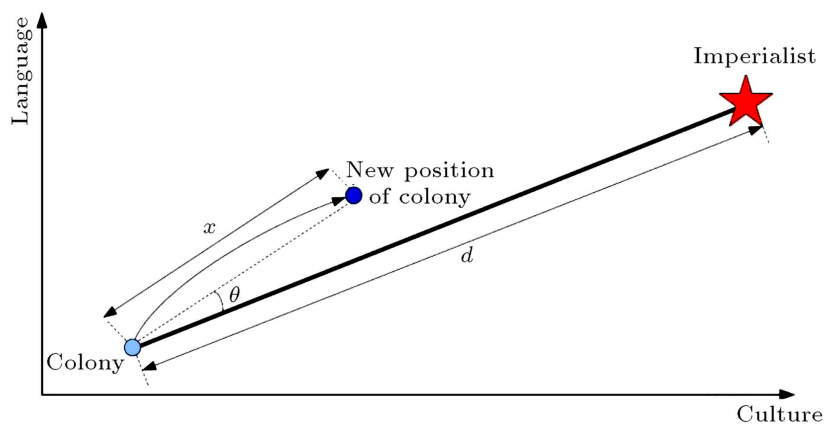

Figure 3. Movement of colonies toward their relevant imperialist in a randomly deviated direction [30].

Figure 3. According to this figure, a colony motion toward the imperialist by $x$ units, where $x$ is a random variable within uniform distribution, is considered to be between 0 and $\beta \times d$ :

$$
x \sim U(0, \beta \times d),
$$

where $\beta$ and $d$ represent a positive number greater than one and the distance between the colony and the imperialist, respectively. Much research was implemented on ICA with $\beta=2$ as an appropriate value $[12,48,51]$. Moreover, $\theta$ is a random variable with a uniform distribution between $-\gamma$ and $\gamma$ :

$$
x \sim U(-\gamma, \gamma)
$$

where $\gamma$ is a control parameter that sets the deviation from the original direction. In order to increase the searching around the imperialist, a random amount of deviation is added to the direction of movement;

\section{- Step 3: Revolution}

Revolution is a powerful strategy that increases the power exploration of the algorithm and helps to escape from local minimums. This strategy is similar to the mutation process in the GA to create diversification in solutions. The probability of revolution in ICA illustrates the percentage of colonies that randomly change their position. The schematic of the revolution strategy is also shown in Figure 4. In this paper, an efficient revolution 


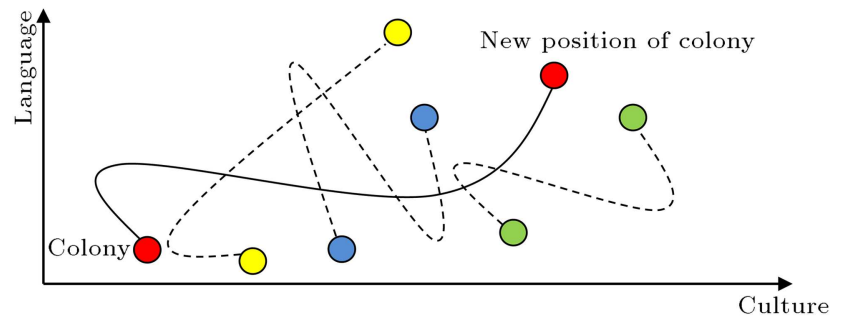

Figure 4. Revolution as a sudden change in socio-political characteristics of a country [51].

strategy is proposed and described in detail in the next session;

- Step 4: Exchanging positions of the imperialist and colony

After applying revolution, a number of colonies may reach a better position (i.e., lower cost) in comparison with their imperialists. In such cases, the colony in each empire moves to the position of that imperialist. This process helps the optimization algorithm avoid getting stuck at locally optimal values;

- Step 5: Total power of an empire

The total power of an empire is defined as the summation of imperialist powers and a percentage of the mean power of its colonies:

$$
\begin{aligned}
T C_{n}= & \operatorname{cost}\left(\text { imperialist }_{n}\right) \\
& +\xi \operatorname{mean}\left\{\operatorname{cost}\left(\text { colonies of empire }_{n}\right)\right\},
\end{aligned}
$$

where $T C_{n}$ is the total cost of $n$th empire, and $\xi$ is a positive small constant less than 1 . The value of 0.1 for $\xi$ has led to good results in most of the implementations [51];

- Step 6: Imperialistic competition Imperialistic competition is a key process in ICA framework in which all empires attempt to take over the possession of colonies from other empires and also control them. This event leads to the reduction of the power of weaker empires, while powerful empires tend to increase their powers. This strategy is formulated by choosing the poorest colony of the poorest empire and making a competition among all empires to possess these colonies. Figure 5 shows this competition, schematically. According to this figure, the more powerful empire is more likely to possess the weakest colony of the weakest empire [33].

To commence the competition, first, the possession probability of each empire is provided as proportionate to the total power of the empire. The normalized total cost is computed by:

$$
N T C_{n}=T C_{n}-\underset{i}{\max }\left\{T C_{i}\right\},
$$

where $T C_{n}$ is the total cost of $n$th empire, and

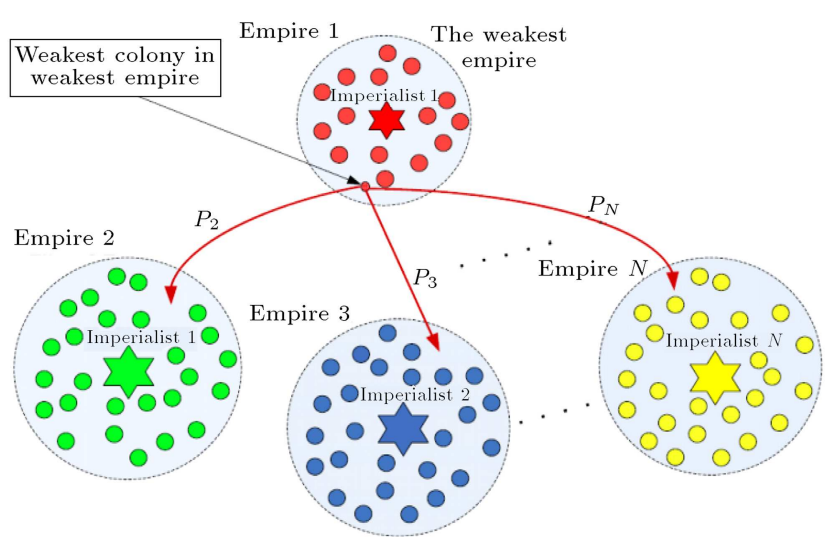

Figure 5. Imperialistic competition [33].

$N T C_{n}$ is its normalized cost. With regard to the normalized total cost, the possession probability of each empire is obtained by:

$$
P_{P_{n}}=\left|\frac{N T C_{n}}{\sum_{i=1}^{N_{\mathrm{imp}}} N T C_{i}}\right| .
$$

To divide colonies among empires, vector $P$ is provided as follows:

$$
P=\left[P_{P_{1}}, P_{P_{2}}, \cdots, P_{P_{N \mathrm{imp}}}\right] .
$$

Then, vector $R$ is created as size $P$ with random numbers within uniform distribution between zero and one.

$$
R=\left[r_{1}, r_{2}, \cdots, r_{N_{\mathrm{imp}}}\right] .
$$

With regard to vectors $R$ and $P$, vector $D$ is determined as follows:

$$
D=P-R=\left[D_{1}, D_{2}, \cdots, D_{N_{\mathrm{imp}}}\right] .
$$

Finally, the highest value of vector $D$ is found and its corresponding index specifies the empire that possesses the colony;

- Step 7: Eliminating the powerless empires When the powerless empires lose all their colonies, they collapse in the imperialistic competition and their colonies are consequently divided among other empires;

\section{- Step 8: Convergence}

ICA is stopped when there is just one empire and all colonies are under the control of that empire. Many stopping criteria for ICA can be found in [51].

\section{An extended ICA}

Since ICA is still in its infancy and intensive studies are needed to improve its performance, many novel operators are proposed $[36,41,49]$. In order to improve the performance of ICA and obtain better solution quality, in this paper, two steps of basic ICA, including assimilation and revolution, are modified. 


\subsection{Modifying colonies' movement}

Considering the movement process of the original ICA, a point out of the colony-imperialistic contacting line can be obtained as shown in Figure 6. This algorithm not only uses different random values, but also utilizes the orthogonal colony-imperialistic contacting line instead of $\theta$ for deviating the colony [46]. To better understand its action, the movement equation is reformed by:

$$
\begin{aligned}
&\{x\}_{\text {new }}=\{x\}_{\text {old }}+\beta \times d \times\{\text { rand }\} \otimes\left\{v_{1}\right\} \\
&+U(-1,1) \times \tan (\theta) \times d \times\left\{v_{2}\right\}, \\
&\left\{v_{1}\right\} \cdot\left\{v_{2}\right\}=0, \quad\left\|\left\{v_{2}\right\}\right\|=1,
\end{aligned}
$$

where $\{$ rand $\}$ is a random vector, sign $\otimes$ indicates an element-by-element multiplication, $\left\{v_{1}\right\}$ is the base vector starting from the previous location of colony and being directed to the imperialistic contacting line; $\left\{v_{2}\right\}$ is perpendicular to $\left\{v_{1}\right\}$. This equation uses both different random values and utilizes the orthogonal colony-imperialistic contacting line instead of $\theta$ to deviate the colony.

\subsection{Modifying revolution strategy}

The second modification is related to revolution strategy. At each iteration (decade), the new revelation is applied as in the following steps:

- Step 1: Set Probability of Revolution $(P R)$ and Revolution Ratio $(R R)$ which depicts the percentage of colonies that randomly change their position and the percentage of the number of variables in each colony which randomly change their value, respectively;

- Step 2: Generate random numbers between zero and one for each colony;

- Step 3: Compare each value of the random numbers with $P R$. If the random numbers are lower than $P R$, the procedure of revolution is performed;

- Step 4: Generate random numbers between zero and one for each variable of colony;

- Step 5: Compare each value of the random numbers with $R R$. If the random number is lower than $R R$, the number of variables is changed;

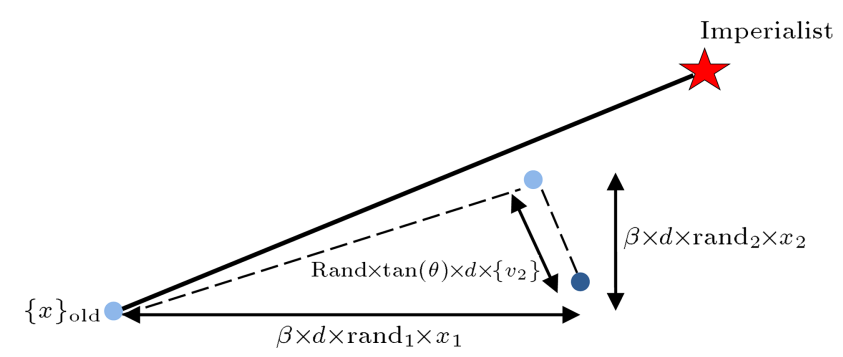

Figure 6. Moving colonies to its next location [46].
- Step 6: Compute step length, $r$, as below:

$$
r=\tanh (z \cdot \mu),
$$

where 'tanh' stands for the hyperbolic tangent function and $z$ shows a random number from a standard normal distribution where the mean value and standard deviation are zero and one, respectively. Finally, $\mu$ is the search parameter which is calculated as a linear combination of initial search parameter $\left(\mu_{0}\right)$ and final search parameter $\left(\mu_{\text {final }}\right)$ as follows:

$$
\mu=\frac{(\text { decade }-1)}{\max \text { decade }} \mu_{\text {final }}+\frac{(\text { max decade }- \text { decade })}{\text { max decade }} \mu_{0} .
$$

According to Eq. (16), when the number of decades of the algorithm is increased, the value of $\mu$ is decreased. This decrease is due to the fact that, in the final decades, the algorithm has most likely obtained near-desired solutions. Therefore, the algorithm requires less variation than in its primary decades. In other words, to make a trade-off between the exploration and exploitation, big initial $\mu$ favors exploration in the initial steps of the search. As the iteration number increases, smaller $\mu$ facilitates the exploitation. Figure 7 shows that the diagram of $\mu$ changes in different decades of the proposed EICA;

- Step 7: Calculate new colony $(y)$ based on Eq. (17) where $u_{k}$ and $l_{k}$ are upper and lower bounds in $k$ th variable.

$$
y_{k}= \begin{cases}y_{k}+r\left(u_{k}-y_{k}\right) & \text { if } r>0 \\ y_{k}+r\left(y_{k}-l_{k}\right) & \text { if } r \leq 0\end{cases}
$$

To clarify the trend of the introduced operator for the evolution process in EICA, the pseudo-code is presented in Figure 8.

According to $P R$ consideration, Figure 9 depicts an example of the new revolution operator in which $R R$ is 0.1 . Therefore, due to the number intended for $R R$, in the uniform vector, the positions that are lower than $R R$ are found. Then, the values of the same positions of the colony vector are

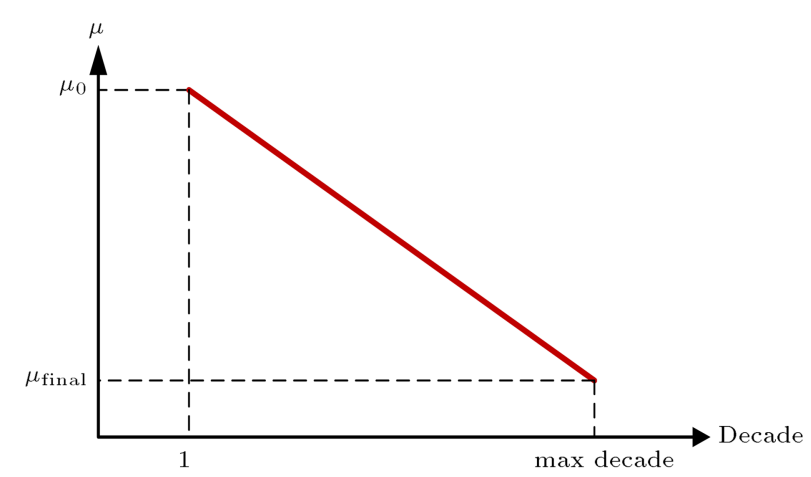

Figure 7. Linear diagram of $\mu$ changes. 


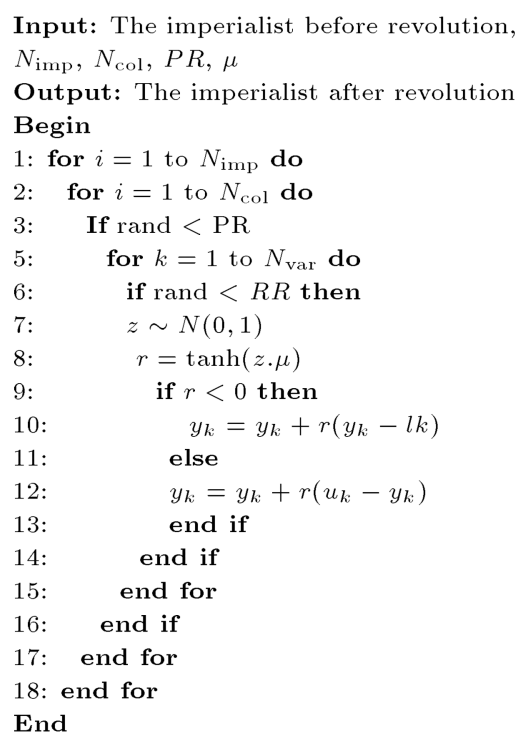

Figure 8. Pseudo-code of novel revolution operator.

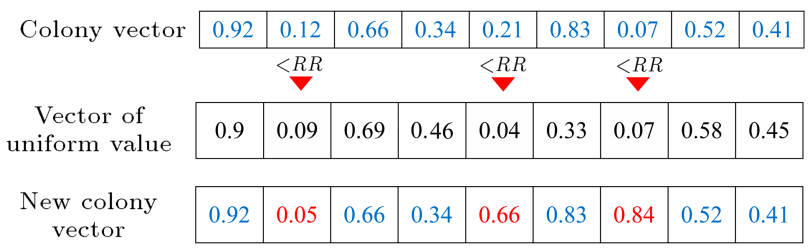

Figure 9. An instance of the proposed revolution procedure.

changed (i.e., the 2 th, 5 th, and 7 th bits of the colony vector). The changes are carried out based on Steps 6 to 8 . Finally, the new colony is obtained.

\section{Prototype classifier based on EICA}

In this paper, an efficient prototype-based classification approach is proposed. The general framework of the proposed EICA-based classifier is presented in Figure 10. The details involved in the general framework are explained in the following subsections.

\subsection{Solution structure}

A one-dimensional array to represent the prototypes in a country in EICA is shown in Figure 11. The country is a vector with length of $S \times K$ where $K$ is the number of clusters and $S$ is the dimensionality (attribute) of the considered datasets. Suppose country $_{i}=$ $\left\{Z_{1}, Z_{2}, \cdots, Z_{K}\right\}$ as $i$ th country $\left(i=1,2, \cdots, N_{\text {pop }}\right)$; then, $Z_{j}=\left\{Z_{j}^{1}, Z_{j}^{2}, \cdots, Z_{j}^{S}\right\}$ is $j$ th prototype for $i$ th country $(j=1,2, \cdots, K)$.

\subsection{The training phase}

Given a dataset representing $K$ classes with $S$ attributes, the goal of EICA in the training phase is to determine $K$ favorable prototypes in $S$-dimensional space for each class according to a specific fitness function and to generate the classification model (i.e., one prototype for each class). To accomplish this goal, the search process to find favorable prototypes in the training set is started by EICA as a global searcher. The procedure of the proposed EICA algorithm for the training process is provided as follows:

- Step 1: Retrieval data. Considering that some of these datasets include missing values, here, the missing values in each attribute are replaced by the average value of the attribute [52-54];

- Step 2: Prepare the needed data. The datasets are divided into the training and test datasets in the ratios of $75 \%$ and $25 \%$. It should be noted that in glass, thyroid, and wine datasets, the dataset classes are in sequential list. Hence, the elements are shuffled;

- Step 3: Some random points are selected from the training set. The fitness value of each country is computed, and the empire is initialized. Three fitness functions, as reported in [37], are picked out, which are explained in Subsection 4.4;

- Step 4: All the colonies are moved and gotten closer to their relevant imperialist (assimilation). This movement is based on the orthogonal colonyimperialistic contacting line, as mentioned in Subsection 3.1 ;

- Step 5: Evaluate cost function;

- Step 6: Change the position of colonies (revolution). This change is based on the proposed revolution, as mentioned in Subsection 3.2;

- Step 7: If there is a colony in an empire that has lower cost than that of the imperialist, then exchange positions of that colony and the imperialist;

- Step 8: Compute the total cost of all empires;

Step 9: Start imperialist competition. Pick the weakest colony (colonies) from the weakest empires and give it (them) to the empires that have more likelihood to possess it (them);

- Step 10: Eliminate the powerless empires that have no colonies;

- Step 11: Termination criteria. The process is repeated and the prototypes positions are updated until the end of the training stage. Then, the best found values for the $K$ prototypes are saved to be used for recall.

Since the training process is overly effective in the performance of the algorithms, an inappropriate training can be converted to a local optimum, and consequently, increase the number of misclassifications. 


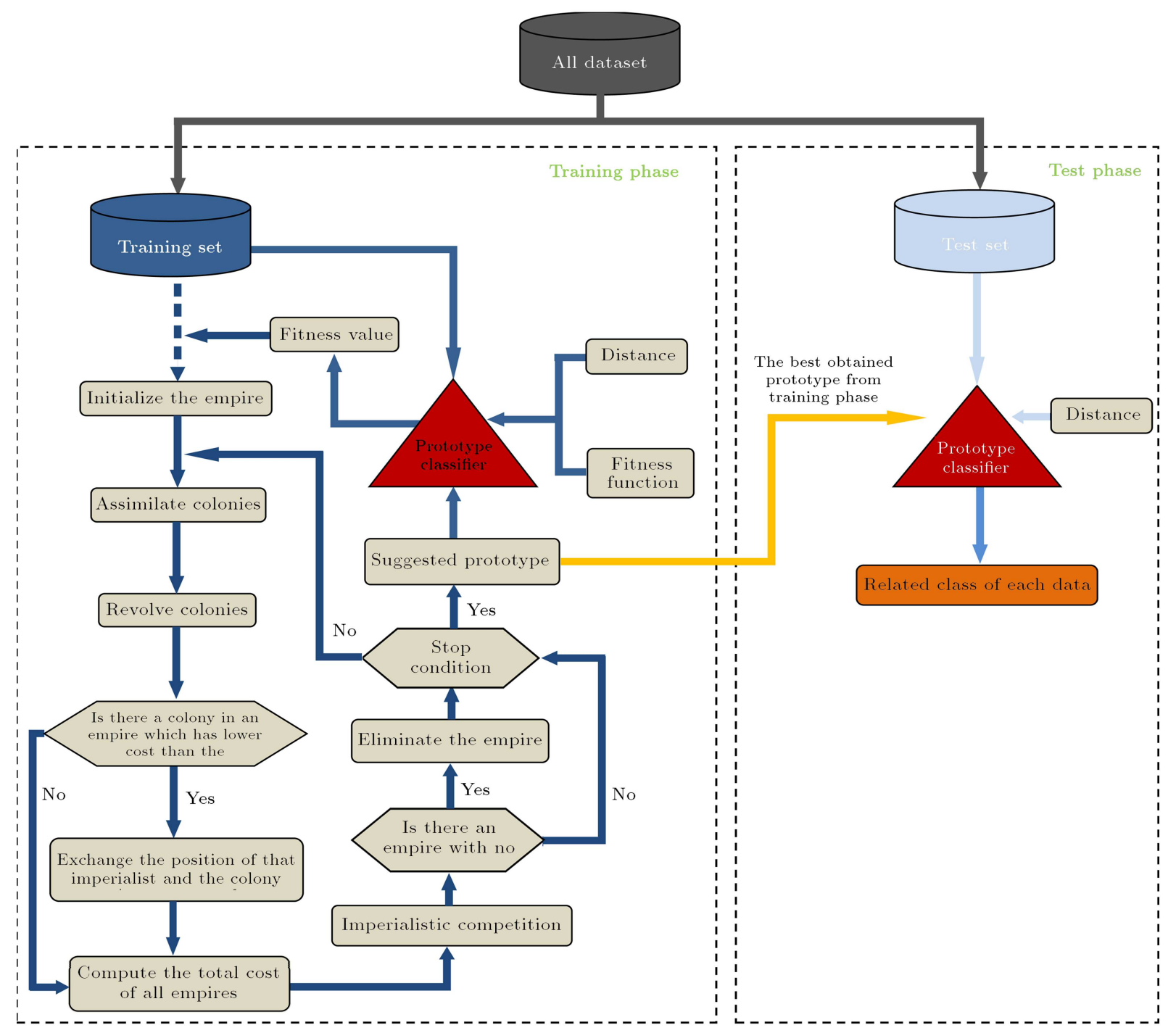

Figure 10. The general framework of the proposed EICA based classifier.

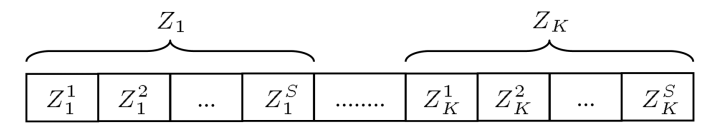

Figure 11. Representation of the prototypes in a country.

\subsection{The test phase}

In this phase, the found prototypes are evaluated with respect to class instances in a test set (the remaining $25 \%$ of dataset) to establish the recognition accuracy. So, for each instance from the test set, its distance from the prototypes is measured. Then, the instance is assigned to the prototype with the closest distance. In this paper, the Euclidian distance algorithm is applied in a way that is similar to the other algorithms.

\subsection{Fitness function}

In this study, three fitness functions as minimization function $\left(f_{1}, f_{2}\right.$, and $\left.f_{3}\right)$ are applied to compare the performance of the proposed EICA with other classification algorithms. For more details about the fitness functions, one can refer to [29]. These three fitness functions are described as follows:

\section{- First fitness functions $\left(f_{1}\right)$}

This fitness function calculates the percentage of incorrectly assigned instances (misclassification) in the training set as follows:

$$
f_{1}=\frac{100}{D_{\text {Train }}} \sum_{j=1}^{D_{\text {Train }}} \delta\left(\vec{x}_{j}\right)
$$

where:

$$
\delta\left(\vec{x}_{j}\right)= \begin{cases}1 & \text { if } C L\left(\vec{x}_{j}\right) \neq C L_{\text {known }}\left(\vec{x}_{j}\right), \\ 0 & \text { otherwise }\end{cases}
$$

where $D_{\text {Train }}$ is the number of training patterns applied to compose the training set, and $C L\left(\vec{x}_{j}\right)$ and 
Table 1. Characteristics of the datasets.

\begin{tabular}{lccccc}
\hline \multicolumn{1}{c}{ Dataset } & $\begin{array}{c}\text { \# of } \\
\text { data set } \\
\text { elements }\end{array}$ & $\begin{array}{c}\text { \# of } \\
\text { training } \\
\text { data }\end{array}$ & $\begin{array}{c}\text { \# of } \\
\text { testing } \\
\text { data }\end{array}$ & $\begin{array}{c}\text { \# of } \\
\text { input } \\
\text { attributes }\end{array}$ & $\begin{array}{c}\text { \# of } \\
\text { classes }\end{array}$ \\
\hline Balance & 625 & 469 & 156 & 4 & 3 \\
Cancer & 569 & 427 & 142 & 30 & 2 \\
Cancer-Int & 699 & 524 & 175 & 9 & 2 \\
Credit & 690 & 518 & 172 & 51 & 2 \\
Dermatology & 366 & 274 & 92 & 34 & 6 \\
Diabetes & 768 & 576 & 192 & 8 & 2 \\
E. coli & 327 & 245 & 82 & 7 & 5 \\
Glass & 214 & 161 & 53 & 9 & 6 \\
Horse & 364 & 273 & 91 & 58 & 3 \\
Iris & 150 & 112 & 38 & 4 & 3 \\
Thyroid & 215 & 162 & 53 & 5 & 3 \\
Wine & 178 & 133 & 45 & 13 & 3 \\
\hline
\end{tabular}

$C L_{\mathrm{known}}\left(\vec{x}_{j}\right)$ are the obtained class for the generic instance $\vec{x}_{j}$ and the class in which generic instance $\vec{x}_{j}$ belongs to database, respectively. Eq. (18) is multiplied by number 100 because the fitness function can vary between $[0,100] . \delta\left(\vec{x}_{j}\right)$ represents the presence (i.e., equal to one) or absence (i.e., equal to zero) of misclassification.

- Second fitness functions $\left(f_{2}\right)$

Fitness function $f_{2}$ denotes the Euclidean distance between generic instance $\vec{x}_{j}$ and the center of the cluster (prototype), belonging to database $\left(\vec{p}_{i}{ }^{C L_{\mathrm{known}}\left(\vec{x}_{j}\right)}\right)$ which is calculated as follows:

$$
f_{2}=\frac{100}{D_{\text {Train }}} \sum_{j=1}^{D_{\text {Train }}} d\left(\vec{x}_{j}, \vec{p}_{i}^{C L_{\text {known }}\left(\vec{x}_{j}\right)}\right) .
$$

Note that the objective value is normalized with $D_{\text {Train }}$;

\section{- Third fitness functions $\left(f_{3}\right)$}

Fitness function $f_{3}$ is divided into two phases. In the first phase, any training instance is assigned to cluster center of closer distance. In the second phase, fitness function $f_{3}$ is obtained as a linear combination of $f_{1}$ and $f_{2}$ as follows:

$$
f_{3}=\frac{1}{2}\left(\frac{f_{1}}{100}+f_{2}\right)
$$

\section{Experimental implementation}

In this section, we first introduce 12 benchmark data sets to compare the performance of methods. Second, we present a performance criterion to measure performance of the methods. Third, we determine proper values of EICA and ICA parameters for the classification problem. Finally, the software and hardware used to implement EICA and ICA algorithms are present.

\subsection{UCI datasets}

For a better assessment of the proposed methodology, 12 real datasets are used. These datasets are obtained from the UCI Machine Learning repository that is used by many researchers to assess their algorithms [50]. The characteristics of these datasets are summarized in Table 1. These datasets have differences in the number of objects, clusters, and attributes. Moreover, as mentioned previously, the datasets are divided into training and test datasets in the ratios of $75 \%$ and $25 \%$. However, using a fixed split may yield biased results [29]. The datasets used in this study are described below:

Balance dataset: This dataset consists of 625 elements which split into 469 for training and 156 for testing. Each instance is classified into three categories:

(I) Balance scale tip to the right;

(II) Balance scale tip to the left;

(III) Be balanced.

The dataset includes four attributes: the left weight, the left distance, the right weight, and the right distance;

Cancer dataset: This is the cancer dataset which is based on the "breast cancer Wisconsin - Diagnostic" 
dataset. It includes classes with a tumor as either benign or malignant. This dataset contains 569 elements with 30 attributes. There are 16 instances containing missing data;

Cancer-Int dataset: This dataset is based on the "breast cancer Wisconsin-Original" dataset. It contains 699 elements, 9 inputs;

Credit dataset: This dataset is based on Australian Credit Card database to assess the applications for credit cards. It contains 690 elements with 14 attributes containing six numeric values and eight discrete formed into 51 input values;

Dermatology dataset: This is the dermatology dataset applied to the case of diagnosis of skin diseases. There are 366 elements, including 34 inputs and 6 classes: psoriasis, seboreic dermatitis, lichen planus, pityriasis rosea, cronic dermatitis, and pityriasis rubra pilaris;

Diabetes dataset: This dataset is the applied diabetes diagnosis which consists of 768 elements from female patients of Pima Indian heritage. The first 576 elements are considered as the training set and the remainder 192 as the test set. There are two classes and eight attributes in the dataset;

E. coli dataset: This dataset originally had 336 elements and eight classes. Three classes are represented with only 2, 2, 5 instances. Therefore, the new dataset is used which contains 327 members, five classes, and seven attributes. In the new dataset, the first 245 elements are considered as the training set and the remainder 82 as the test set;

Glass dataset: This dataset contains samples from six different types of glass: building windows float processed, vehicle windows, building windows non-float processed, tableware, and headlamps. There are 214 elements with nice inputs which are split into 161 for training and 53 for testing;

Horse dataset: Horse dataset is used to predict the fate of a horse with colic and to classify whether the horse will survive, die, or be euthanized. The dataset is created based on Horse Colic data with 364 patterns, each of which has 58 inputs from 27 attributes and three outputs. In this dataset, $30 \%$ of the values are missing;

Iris dataset: The Iris flower dataset or Fisher's Iris dataset is a multivariate dataset introduced by Sir Ronald Fisher as an example of discriminate analysis.
This dataset contains three categories of 50 objects where each category refers to a type of iris plant. In the iris dataset, there are 150 instances with four attributes which are sepal length in centimeter $(\mathrm{cm})$, sepal width in $\mathrm{cm}$, petal length in $\mathrm{cm}$, and petal width in $\mathrm{cm}$, respectively;

Thyroid dataset: This dataset contains three categories of human thyroid diseases, namely, euthyroidism, hypothyroidism, and hyperthyroidism. This dataset has 215 elements with five attributes. These are the T3-resin uptake test, total serum thyroxin as measured by the isotopic displacement method, total serum triiodothyronine as measured by radioimmuno assay, basal thyroidstimulating hormone as measured by radioimmuno of $200 \mathrm{mg}$ of thyrotropin releasinghormone, and the basal value;

Wine dataset: These data are the results of a chemical analysis of wines grown in the same region in Italy extracted from three different cultivars. This dataset contains 178 elements by 13 attributes. The attributes are alcohol, malic acid, ash, alcalinity of ash, magnesium, total phenols, flavanoids, nonflavanoid phenols, proanthocyanins, color intensity, hue, OD280/OD315 of diluted wines and proline.

\subsection{Performance criteria}

The performance of the algorithms is measured by Classification Error Percentage (CEP) criterion. The percentage of incorrectly classified patterns of the test datasets are shown as follows:

$$
\mathrm{CEP}=\frac{\text { number of misclassified examples }}{\text { total size of test dataset }} \times 100 \text {. }
$$

According to this criterion, each pattern is classified by assigning it to the class whose distance is closest to the prototype of the clusters. Then, the classified output is compared with the desired output. If they are not exactly the same, the pattern is separated as misclassified. The CEP creation is determined and reported for all test data. It should be noted that the CPU times of the techniques, as a competitive benchmark, are not considered.

\subsection{Parameter setting}

Determining proper values for the optimization algorithms parameters has played a key role in its performance. For tuning EICA and ICA for the classification problem, extensive experiments were conducted with differing sets of parameters. At the end, the following sets were found to be effective in terms of solutions quality: the maximum number of iterations ( $\max$ decade), the number of countries $\left(N_{\text {pop }}\right)$, the number of imperialists $\left(N_{\text {imp }}\right)$, Probability of Revolution $(P R)$, Revolution Ratio $(R R)$, initial search parameter $\left(\mu_{0}\right)$, 
Table 2. Values of parameters of EICA and ICA algorithms.

\begin{tabular}{lcclcc}
\hline \multicolumn{2}{c}{ EICA } & & & \multicolumn{2}{c}{ ICA } \\
\cline { 1 - 2 } \cline { 5 - 5 } Parameters & Value & & Parameters & Value \\
\hline$N_{\text {pop }}$ & 100 & & maxdecade & 100 \\
$N_{\text {imp }}$ & 100 & & $N_{\text {pop }}$ & 200 \\
$P R$ & 5 & & $N_{\text {imp }}$ & 5 \\
$R R$ & 0.1 & & $P R$ & 0.1 \\
$\mu_{0}$ & 0.3 & & $R R$ & 0.3 \\
$\mu_{\text {final }}$ & 0.2 & & \\
\hline
\end{tabular}

and final search parameter $\left(\mu_{\text {final }}\right)$. The values of EICA and ICA parameters are represented in Table 2.

\subsection{Hardware and software specifications}

EICA and ICA algorithms were implemented in MATLAB software (version 7.10.0.499, R2010a) and run on a $1.6 \mathrm{GHz}$ laptop with $6 \mathrm{~GB}$ of RAM under a 64 -bit Windows operating system.

\section{Experimental results and discussion}

The experimental part of the paper is structured in five subsections. First, in order to show the improvement obtained using EICA compared to the original ICA [33], we compare EICA with ICA. Second, we compare the performance of EICA with that of well-developed algorithms in classification (i.e., PSO [29], ABC [30], FA [31], GSA [28], and GGSA [32]). Third, the performance of EICA is compared against those of the other nine classification techniques well known in literature reported in [15] as: Bayes Net [55], MultiLayer Perception artificial neural network (MLP) [56], Radial Basis Function artificial neural network (RBF) [57], K Star [58], Bagging [59], Multi Boost AB [60], Naive Bayes Bayes Tree (NB Tree) [61], ripple down rule (Ridor) [62], and Voting Feature Interval (VFI) [63]. Fourth, one additional comparison between all classification methods is considered. Finally, a discussion section from the results obtained is considered.

\subsection{Comparison between EICA and ICA}

In order to show the improvement obtained using EICA compared to ICA, the 12 datasets are used once more. The comparison of results for each dataset is based on the average CEP obtained after more than 20 different simulations. For any dataset, the best results (i.e., lowest) based on $f_{1}, f_{2}$, and $f_{3}$ are indicated in bold type. The results are summarized in Tables 3-5. As it can be easily seen in these tables, EICA performs consistently better than ICA in most of the datasets. For example, the results shown in Table 3 indicate that the proposed EICA algorithm outperforms the basic ICA algorithm for 11 datasets. Compared to the basic ICA classifier, the proposed EICA algorithm has respectively improved the classification accuracy rates of the Horse dataset by $12.55 \%$, the Glass dataset by $22.02 \%$, the Dermatology dataset by $25.46 \%$, and the Cancer dataset by $22.60 \%$. These results are also depicted in Figure 12.

Figure 13 provides more insight into the convergence rate of EICA and ICA algorithms for the best solutions to Iris dataset. Simulation results of the figure show that EICA algorithm converges faster than

Table 3. Average classification error percentages of EICA and ICA based on $f_{1}$.

\begin{tabular}{|c|c|c|c|c|c|c|}
\hline \multirow{3}{*}{ Dataset } & \multicolumn{6}{|c|}{ Classifier } \\
\hline & \multicolumn{3}{|c|}{ EICA } & \multicolumn{2}{|l|}{ ICA } & \multirow[b]{2}{*}{ s.t.d } \\
\hline & Average & Best & s.t.d & Average & Best & \\
\hline Balance & 11.76 & 11.53 & 0.17 & 13.24 & 12.82 & 0.32 \\
\hline Cancer & 5.24 & 4.92 & 0.48 & 5.01 & 4.98 & 0.11 \\
\hline Cancer-Int & 0.57 & 0.57 & 0 & 2.45 & 1.71 & 0.66 \\
\hline Credit & 14.44 & 13.95 & 0.65 & 16.50 & 16.27 & 0.37 \\
\hline Dermatology & 13.64 & 13.04 & 0.82 & 18.30 & 17.39 & 0.40 \\
\hline Diabetes & 21.58 & 21.35 & 0.42 & 22.81 & 21.87 & 0.21 \\
\hline E. coli & 6.64 & 6.09 & 0.92 & 8.40 & 7.31 & 0.38 \\
\hline Glass & 29.71 & 28.30 & 2.28 & 38.10 & 35.84 & 1.49 \\
\hline Horse & 35.60 & 35.16 & 0.74 & 40.71 & 38.04 & 1.32 \\
\hline Iris & 0 & 0 & 0 & 0 & 0 & 0 \\
\hline Thyroid & 0 & 0 & 0 & 5.31 & 3.77 & 0.74 \\
\hline Wine & 0 & 0 & 0 & 2.73 & 2.22 & 0.97 \\
\hline Average & 11.60 & & & 14.46 & & \\
\hline
\end{tabular}


Table 4. Average classification error percentages of EICA and ICA based on $f_{2}$.

\begin{tabular}{|c|c|c|c|c|c|c|}
\hline \multirow{3}{*}{ Dataset } & \multicolumn{6}{|c|}{ Classifier } \\
\hline & \multicolumn{3}{|c|}{ EICA } & \multicolumn{2}{|l|}{ ICA } & \multirow[b]{2}{*}{ s.t.d } \\
\hline & Average & Best & s.t.d & Average & Best & \\
\hline Balance & 9.29 & 8.97 & 0.53 & 11.45 & 10.89 & 0.21 \\
\hline Cancer & 2.81 & 2.81 & 0 & 5.15 & 4.22 & 0.49 \\
\hline Cancer-Int & $\mathbf{0}$ & 0 & 0 & 2.12 & 1.71 & 0.44 \\
\hline Credit & 14.21 & 13.95 & 0.44 & 16.17 & 15.69 & 0.33 \\
\hline Dermatology & 13.31 & 13.04 & 0.59 & 18.12 & 16.30 & 0.88 \\
\hline Diabetes & 21.22 & 20.83 & 0.69 & 24.60 & 23.43 & 0.78 \\
\hline E. coli & 5.60 & 4.87 & 1.50 & 7.11 & 6.09 & 1.01 \\
\hline Glass & 26.13 & 24.52 & 2.54 & 30.79 & 30.18 & 0.53 \\
\hline Horse & 33.43 & 32.96 & 0.79 & 38.71 & 38.04 & 0.43 \\
\hline Iris & $\mathbf{0}$ & 0 & 0 & $\mathbf{0}$ & 0 & 0 \\
\hline Thyroid & $\mathbf{0}$ & 0 & 0 & 3.01 & 1.88 & 1.18 \\
\hline Wine & 0 & 0 & 0 & 2.64 & 2.22 & 0.29 \\
\hline Average & 10.50 & & & 13.32 & & \\
\hline
\end{tabular}

Table 5. Average classification error percentages of EICA and ICA based on $f_{3}$.

\begin{tabular}{|c|c|c|c|c|c|c|}
\hline \multirow{3}{*}{ Dataset } & \multicolumn{6}{|c|}{ Classifier } \\
\hline & \multicolumn{3}{|c|}{ EICA } & \multicolumn{2}{|l|}{ ICA } & \multirow[b]{2}{*}{ s.t.d } \\
\hline & Average & Best & s.t.d & Average & Best & \\
\hline Balance & 10.44 & 10.25 & 0.42 & 12.00 & 11.53 & 0.43 \\
\hline Cancer & 3.69 & 3.52 & 0.38 & 4.88 & 4.22 & 0.62 \\
\hline Cancer-Int & 0 & 0 & 0 & 2.55 & 2.28 & 0.24 \\
\hline Credit & 14.30 & 13.95 & 0.47 & 16.20 & 15.69 & 0.71 \\
\hline Dermatology & 13.42 & 13.04 & 0.63 & 17.92 & 16.30 & 1.08 \\
\hline Diabetes & 20.37 & 20.31 & 0.15 & 23.05 & 21.87 & 0.81 \\
\hline E. coli & 6.52 & 6.09 & 0.81 & 6.23 & 6.11 & 0.21 \\
\hline Glass & 25.37 & 24.52 & 1.43 & 37.98 & 37.73 & 0.18 \\
\hline Horse & 33.29 & 32.96 & 0.69 & 39.01 & 38.04 & 0.33 \\
\hline Iris & 0 & 0 & 0 & 3.11 & 2.63 & 0.47 \\
\hline Thyroid & 0 & 0 & 0 & 3.54 & 1.88 & 1.13 \\
\hline Wine & 0 & 0 & 0 & 1.88 & 0 & 0.41 \\
\hline Average & 10.62 & & & 14.02 & & \\
\hline
\end{tabular}

ICA algorithm. EICA algorithm converges in about 50 iterations to the global optimum, while ICA converges to the global optimum after 90 iterations.

In order to illustrate the significant differences among the results obtained by the proposed EICA and ICA, statistical testing is used. Because the obtained results may present neither normal distribution nor homogeneity of variance, we suggest using non-parametric tests [64]. Specifically, the Friedman test [65], as well as the Iman-Davenport test [66], is employed to determine whether there are significant differences in the results of clustering algorithms or not.
Tables $6-8$ present the $p$-value computed by the Friedman test and the Iman-Davenport test, which confirm the existence of significant differences between the performances of EICA and ICA algorithms.

\subsection{Comparison between EICA and well-developed algorithms}

For a better comparison, the results of EICA and other optimization algorithms based on $f_{1}, f_{2}$, and $f_{3}$ are shown in Tables 9-11. For any dataset, the best results are indicated in bold type. The reason is that the number of classifiers considered for comparison using 


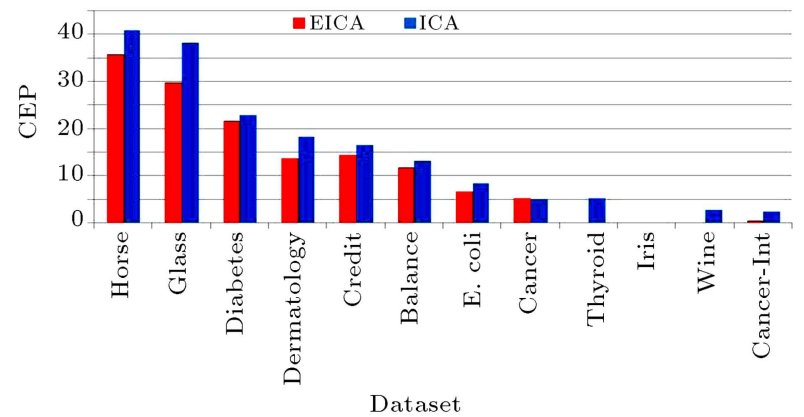

(a)

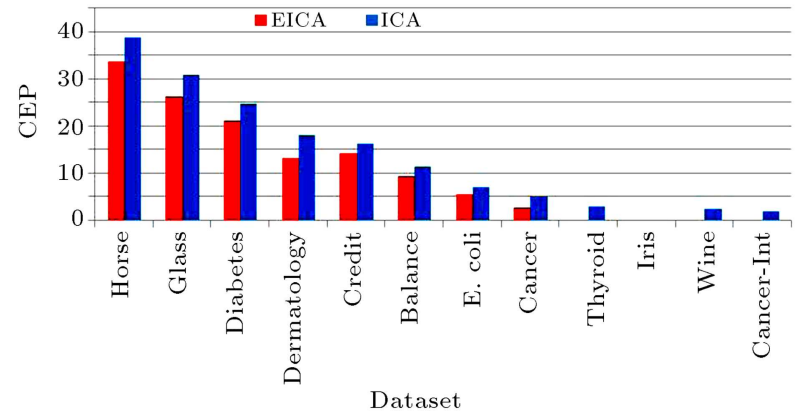

(b)

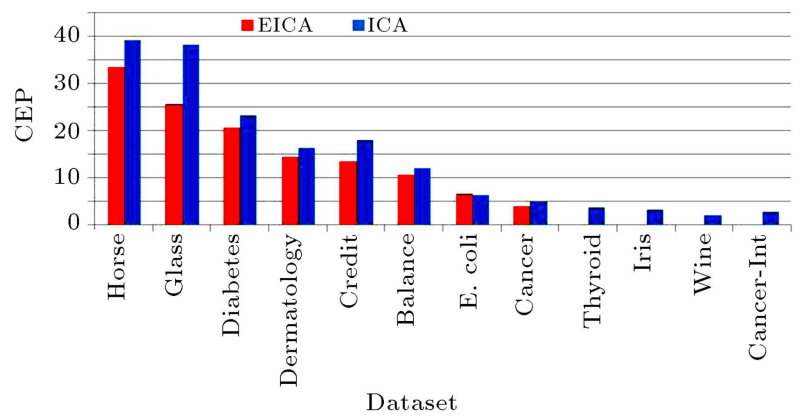

(c)

Figure 12. Comparison between EICA and ICA: (a) Based on $f_{1}$, (b) based on $f_{2}$, and (c) based on $f_{3}$.

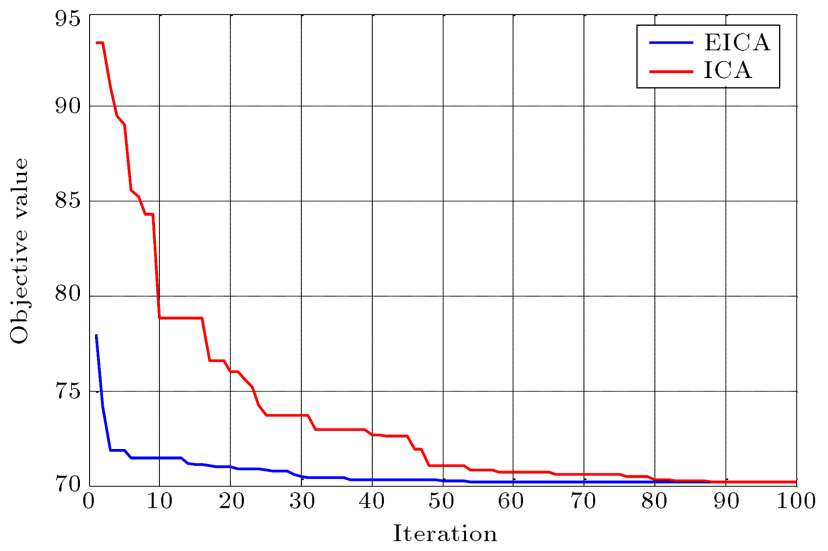

Figure 13. Convergence rate of EICA and ICA algorithms for the best solutions in Iris dataset.

three fitness functions in the tables is not the same, which is due to the fact that there are no results on fitness functions $f_{1}$ and $f_{3}$ in [30-32].
Table 6. Results of Friedman and Iman-Davenport's tests [64] based on $f_{1}$.

\begin{tabular}{lccl}
\hline \multicolumn{1}{c}{ Method } & $\begin{array}{c}\text { Statistical } \\
\text { value }\end{array}$ & $\boldsymbol{p}$-value & Hypothesis \\
\hline Friedman & 6.75 & 0.009 & Rejected \\
Iman-Davenport & 14.142 & 0.003 & Rejected \\
\hline
\end{tabular}

Table 7. Results of Friedman and Iman-Davenport's tests [64] based on $f_{2}$.

\begin{tabular}{lccl}
\hline \multicolumn{1}{c}{ Method } & $\begin{array}{c}\text { Statistical } \\
\text { value }\end{array}$ & $\boldsymbol{p}$-value & Hypothesis \\
\hline Friedman & 10.08 & 0.001 & Rejected \\
Iman-Davenport & 57.75 & 0.000 & Rejected \\
\hline
\end{tabular}

Table 8. Results of Friedman and Iman-Davenport's tests [64] based on $f_{3}$.

\begin{tabular}{lccl}
\hline \multicolumn{1}{c}{ Method } & $\begin{array}{c}\text { Statistical } \\
\text { value }\end{array}$ & $\boldsymbol{p}$-value & Hypothesis \\
\hline Friedman & 8.33 & 0.004 & Rejected \\
Iman-Davenport & 24.967 & 0.000 & Rejected \\
\hline
\end{tabular}

Table 9. Average classification error percentages of PSO, GSA, and EICA based on $f_{1}$.

\begin{tabular}{lccc}
\hline \multirow{2}{*}{ Dataset } & \multicolumn{3}{c}{ Classifier } \\
\cline { 2 - 4 } & PSO & GSA & EICA \\
\hline Balance & 25.47 & 12.67 & $\mathbf{1 1 . 7 6 0 8}$ \\
Cancer & 5.80 & $\mathbf{1 . 5 3}$ & 5.2464 \\
Cancer-Int & 2.87 & 0.97 & $\mathbf{0 . 5 7 1 4}$ \\
Credit & 22.96 & $\mathbf{1 4 . 3 6}$ & 14.4447 \\
Dermatology & 5.76 & $\mathbf{3 . 8 0}$ & 13.6413 \\
Diabetes & 22.50 & $\mathbf{2 1 . 4 0}$ & 21.5883 \\
E. coli & 14.63 & 7.37 & $\mathbf{6 . 6 4 6 3}$ \\
Glass & 40.18 & 33.01 & $\mathbf{2 9 . 7 1 6 9}$ \\
Horse & 40.98 & $\mathbf{3 3 . 9 1}$ & 35.6043 \\
Iris & 2.63 & 1.84 & $\mathbf{0}$ \\
Thyroid & 5.55 & 3.14 & $\mathbf{0}$ \\
Wine & 2.22 & 0.90 & $\mathbf{0}$ \\
\hline
\end{tabular}

In Table 9, EICA results are the best in comparison with the other algorithms based on fitness function $f_{1}$. The proposed method obtained good results with the dataset Balance, Cancer-Int, E. coli, Glass, Iris, Thyroid, and wine and outperformed other classifiers.

In Table 10, the quality obtained by the proposed algorithm based on fitness function $f_{2}$ is much better than the other algorithms in Balance and Glass datasets.

Finally, according to Table 11, in $75 \%$ of datasets (i.e., Balance, Cancer-Int, Credit, Diabetes, E. coli, Glass, Iris, Thyroid, and Wine), EICA algorithm 
Table 10. Average classification error percentages of PSO, GSA, ABC, FA, GGSA, and EICA based on $f_{2}$.

\begin{tabular}{lcccccc}
\hline \multirow{2}{*}{ Dataset } & \multicolumn{6}{c}{ Classifier } \\
\cline { 2 - 7 } & PSO & GSA & ABC & FA & GGSA & EICA \\
\hline Balance & 13.24 & 18.88 & 15.38 & 14.1 & 10.46 & $\mathbf{9 . 2 9 3 1}$ \\
Cancer & 3.49 & 4.16 & 2.81 & $\mathbf{1 . 0 6}$ & 1.13 & 2.8169 \\
Cancer-Int & 2.75 & $\mathbf{0}$ & $\mathbf{0}$ & $\mathbf{0}$ & $\mathbf{0}$ & $\mathbf{0}$ \\
Credit & 22.19 & $\mathbf{1 0 . 9 4}$ & 13.37 & 12.79 & 11.68 & 14.2150 \\
Dermatology & 19.67 & 5.27 & 5.43 & 5.43 & $\mathbf{2 . 8 2}$ & 13.3152 \\
Diabetes & 23.22 & 23.59 & 22.39 & 21.88 & $\mathbf{1 6 . 3 5}$ & 21.2239 \\
E. coli & 23.65 & 11.22 & 13.41 & 8.54 & $\mathbf{3 . 1 1}$ & 5.6091 \\
Glass & 40.18 & 32.07 & 41.50 & 37.73 & 26.57 & $\mathbf{2 6 . 1 3 2 0}$ \\
Horse & 37.69 & 33.31 & 38.26 & $\mathbf{3 2 . 9 7}$ & $\mathbf{2 9 . 9}$ & 33.4423 \\
Iris & 3.68 & 2.63 & $\mathbf{0}$ & $\mathbf{0}$ & $\mathbf{0}$ & $\mathbf{0}$ \\
Thyroid & 6.66 & 1.85 & 3.77 & $\mathbf{0}$ & 0.21 & $\mathbf{0}$ \\
Wine & 6.22 & 2.27 & $\mathbf{0}$ & $\mathbf{0}$ & $\mathbf{0}$ & $\mathbf{0}$ \\
\hline
\end{tabular}

Table 11. Average classification error percentages of PSO, GSA, and EICA based on $f_{3}$.

\begin{tabular}{lccc}
\hline \multirow{2}{*}{ Dataset } & \multicolumn{3}{c}{ Classifier } \\
\cline { 2 - 4 } & PSO & GSA & EICA \\
\hline Balance & 13.12 & 12.92 & $\mathbf{1 0 . 4 4 8 7}$ \\
Cancer & 3.49 & $\mathbf{1 . 3 9}$ & 3.6971 \\
Cancer-Int & 2.64 & 0.91 & $\mathbf{0}$ \\
Credit & 18.77 & 14.42 & $\mathbf{1 4 . 3 0 2 2}$ \\
Dermatology & 6.08 & $\mathbf{4 . 1 2}$ & 13.4239 \\
Diabetes & 21.77 & 20.88 & $\mathbf{2 0 . 3 7 7 0}$ \\
E. coli & 13.90 & 7.31 & $\mathbf{6 . 5 2 4 1}$ \\
Glass & 38.67 & 31.89 & $\mathbf{2 5 . 3 7 7 3}$ \\
Horse & 35.16 & $\mathbf{3 3 . 1 5}$ & 33.2960 \\
Iris & 5.26 & 2.89 & $\mathbf{0}$ \\
Thyroid & 3.88 & 3.14 & $\mathbf{0}$ \\
Wine & 2.88 & 1.58 & $\mathbf{0}$ \\
\hline
\end{tabular}

has the best performance of the other optimization algorithms.

\subsubsection{Comparison between EICA and GGSA algorithms}

The results reported in Table 10 show that EICA obtained acceptable results in comparison with GGSA; GGSA gets better results in the case of the datasets Cancer, Credit, Dermatology, Diabetes, and E. coli, whereas both EICA and GGSA get the same results in the case of Iris, Wine, and Cancer-Int datasets.

To be able to make a good comparison between EICA and GGSA algorithms, we conduct Friedman's and Iman-Davenport's tests. Table 12 shows the resultant $p$-values when comparing among EICA and GGSA on the 12 benchmark datasets. Based on the
Table 12. Results of Friedman and Iman-Davenport's tests based on $f_{2}$.

\begin{tabular}{lccl}
\hline \multicolumn{1}{c}{ Method } & $\begin{array}{c}\text { Statistical } \\
\text { value }\end{array}$ & $\boldsymbol{p}$-value & Hypothesis \\
\hline Friedman & 0.75 & 0.386 & Not rejected \\
Iman-Davenport & 0.7333 & 0.4101 & Not rejected \\
\hline
\end{tabular}

results, it can be seen that there is no statistical significance between EICA and GGSA algorithms.

\subsection{Comparison between EICA and well-known classification techniques}

Table 13 shows the results of EICA and nine wellknown classification techniques in the literature in each of the 12 databases with respect to the average CEP. For any dataset, the best results are indicated in bold type. As can be seen, in $58 \%$ of dataset (i.e., Balane, Cancer-Int, Diabetes, E.coli, Iris, Thyroid, and Wine), EICA algorithm has the best performance compared to the classification techniques.

\subsection{General comparisons}

Table 14 shows the average of CEP obtained by all algorithms in each dataset, and their corresponding rank is shown in bracket. For any dataset, the best results are indicated in bold type. Note that we consider the best sum average of CEP found algorithms based on $f_{1}, f_{2}$, and $f_{3}$ as a criterion for the comparison being fair. For example, when we mention EICA performance, we will make reference to that of EICA based on $f_{2}$.

Researchers were interested to find out which algorithm, out of 16 algorithms, have superior classification accuracy. Hence, two comparisons for evaluation between all algorithms can be considered. The first comparison is based on average CEP in all datasets; 
Table 13. Average classification error percentages of EICA and the classifiers given in [29].

\begin{tabular}{|c|c|c|c|c|c|c|c|c|c|c|}
\hline \multirow[b]{2}{*}{ Dataset } & \multicolumn{10}{|c|}{ Classifier } \\
\hline & $\begin{array}{c}\text { Bayes } \\
\text { net }\end{array}$ & $\begin{array}{l}\text { MLP } \\
\text { ANN }\end{array}$ & RBF & $\begin{array}{c}\text { K } \\
\text { Star }\end{array}$ & Bagging & $\begin{array}{l}\text { Multi } \\
\text { boost }\end{array}$ & $\begin{array}{l}\text { NB } \\
\text { tree }\end{array}$ & Ridor & VFI & EICA \\
\hline Balance & 19.74 & 9.29 & 33.61 & 10.25 & 14.77 & 24.20 & 19.74 & 20.63 & 38.85 & 9.29 \\
\hline Cancer & 4.19 & 2.93 & 20.27 & 2.44 & 4.47 & 5.59 & 7.69 & 6.36 & 7.34 & 2.81 \\
\hline Cancer-Int & 3.42 & 5.25 & 8.17 & 4.57 & 3.93 & 5.14 & 5.71 & 5.48 & 5.71 & 0 \\
\hline Credit & 12.13 & 13.81 & 43.29 & 19.18 & 10.68 & 12.71 & 16.18 & 12.65 & 16.47 & 14.21 \\
\hline Dermatology & 1.08 & 3.26 & 34.66 & 4.66 & 3.47 & 53.26 & 1.08 & 7.92 & 7.60 & 13.31 \\
\hline Diabetes & 25.52 & 29.16 & 39.16 & 34.05 & 26.87 & 27.08 & 25.52 & 29.31 & 34.37 & 21.22 \\
\hline E. coli & 17.07 & 13.53 & 24.38 & 18.29 & 15.36 & 31.70 & 20.7 & 17.07 & 17.07 & 5.60 \\
\hline Glass & 29.62 & 28.51 & 44.44 & 17.58 & 25.36 & 53.70 & 24.07 & 31.66 & 41.11 & 26.13 \\
\hline Horse & 30.76 & 32.19 & 38.46 & 35.71 & 30.32 & 38.46 & 31.86 & 31.86 & 41.75 & 33.44 \\
\hline Iris & 2.63 & 0.00 & 9.99 & 0.52 & 0.26 & 2.63 & 2.63 & 0.52 & 0.00 & 0 \\
\hline Thyroid & 6.66 & 1.85 & 5.55 & 13.32 & 14.62 & 7.40 & 11.11 & 8.51 & 11.11 & 0 \\
\hline Wine & 0.00 & 1.33 & 2.88 & 3.99 & 2.66 & 17.77 & 2.22 & 5.10 & 5.77 & 0 \\
\hline
\end{tabular}

Table 14. Achieved results in terms of average classification error percentages of the classifiers given in [29] and the five classifiers PSO, GSA, ABC, FA, ICA, and GGSA.

\begin{tabular}{|c|c|c|c|c|c|c|c|c|c|c|c|c|c|c|c|c|}
\hline \multirow[b]{2}{*}{ Dataset } & \multicolumn{16}{|c|}{ Classifier } \\
\hline & $\begin{array}{c}\text { Bayes } \\
\text { net }\end{array}$ & $\begin{array}{l}\text { MLP } \\
\text { ANN }\end{array}$ & RBF & $\begin{array}{c}\text { K } \\
\text { Star }\end{array}$ & Bagging & $\begin{array}{l}\text { Multi } \\
\text { boost }\end{array}$ & $\begin{array}{l}\text { NB } \\
\text { tree }\end{array}$ & Ridor & VFI & PSO & GSA & $\mathrm{ABC}$ & FA & ICA & GGSA & EICA \\
\hline Balance & $\begin{array}{c}19.74 \\
(11)\end{array}$ & $\begin{array}{c}9.29 \\
(1)\end{array}$ & $\begin{array}{c}33.61 \\
(15)\end{array}$ & $\begin{array}{c}10.25 \\
(3)\end{array}$ & $\begin{array}{c}14.77 \\
(9)\end{array}$ & $\begin{array}{c}24.20 \\
(14)\end{array}$ & $\begin{array}{c}19.74 \\
(11)\end{array}$ & $\begin{array}{c}20.63 \\
(13)\end{array}$ & $\begin{array}{c}38.85 \\
(16)\end{array}$ & $\begin{array}{c}13.12 \\
(7)\end{array}$ & $\begin{array}{c}12.92 \\
(6)\end{array}$ & $\begin{array}{c}15.38 \\
(10)\end{array}$ & $\begin{array}{c}14.1 \\
(8)\end{array}$ & $\begin{array}{c}11.45 \\
(5)\end{array}$ & $\begin{array}{c}10.46 \\
(4)\end{array}$ & $\begin{array}{l}9.29 \\
(1)\end{array}$ \\
\hline Cancer & $\begin{array}{l}4.19 \\
(9)\end{array}$ & $\begin{array}{c}2.93 \\
(7)\end{array}$ & $\begin{array}{c}20.27 \\
(16)\end{array}$ & $\begin{array}{c}2.44 \\
(4)\end{array}$ & $\begin{array}{l}4.47 \\
(10)\end{array}$ & $\begin{array}{l}5.59 \\
(12)\end{array}$ & $\begin{array}{l}7.69 \\
(15)\end{array}$ & $\begin{array}{l}6.36 \\
(13)\end{array}$ & $\begin{array}{l}7.34 \\
(14)\end{array}$ & $\begin{array}{c}3.49 \\
(8)\end{array}$ & $\begin{array}{c}1.39 \\
(3)\end{array}$ & $\begin{array}{c}2.81 \\
(5)\end{array}$ & $\begin{array}{c}1.06 \\
(1)\end{array}$ & $\begin{array}{l}5.15 \\
(11)\end{array}$ & $\begin{array}{c}1.13 \\
(2)\end{array}$ & $\begin{array}{l}2.81 \\
(5)\end{array}$ \\
\hline Cancer-Int & $\begin{array}{c}3.42 \\
(8)\end{array}$ & $\begin{array}{l}5.25 \\
(12)\end{array}$ & $\begin{array}{l}8.17 \\
(16)\end{array}$ & $\begin{array}{l}4.57 \\
(10)\end{array}$ & $\begin{array}{c}3.93 \\
(9)\end{array}$ & $\begin{array}{l}5.14 \\
(11)\end{array}$ & $\begin{array}{l}5.71 \\
(14)\end{array}$ & $\begin{array}{l}5.48 \\
(13)\end{array}$ & $\begin{array}{l}5.71 \\
(14)\end{array}$ & $\begin{array}{l}2.64 \\
(7)\end{array}$ & $\begin{array}{c}0.91 \\
(5)\end{array}$ & $\begin{array}{c}0 \\
(1)\end{array}$ & $\begin{array}{c}0 \\
(1)\end{array}$ & $\begin{array}{c}2.12 \\
(6)\end{array}$ & $\begin{array}{c}0 \\
(1)\end{array}$ & $\begin{array}{c}0 \\
(1)\end{array}$ \\
\hline Credit & $\begin{array}{c}12.13 \\
(3)\end{array}$ & $\begin{array}{c}13.81 \\
(8)\end{array}$ & $\begin{array}{c}43.29 \\
(16)\end{array}$ & $\begin{array}{c}19.18 \\
(15)\end{array}$ & $\begin{array}{c}10.68 \\
(1)\end{array}$ & $\begin{array}{c}12.71 \\
(5)\end{array}$ & $\begin{array}{c}16.18 \\
(12)\end{array}$ & $\begin{array}{c}12.65 \\
(4)\end{array}$ & $\begin{array}{c}16.47 \\
(13)\end{array}$ & $\begin{array}{c}18.77 \\
(14)\end{array}$ & $\begin{array}{c}14.42 \\
(10)\end{array}$ & $\begin{array}{c}13.37 \\
(7)\end{array}$ & $\begin{array}{c}12.79 \\
(6)\end{array}$ & $\begin{array}{c}16.17 \\
(11)\end{array}$ & $\begin{array}{c}11.68 \\
(2)\end{array}$ & $\begin{array}{c}14.21 \\
(9)\end{array}$ \\
\hline Dermatology & $\begin{array}{c}1.08 \\
(1)\end{array}$ & $\begin{array}{c}3.26 \\
(4)\end{array}$ & $\begin{array}{c}34.66 \\
(15)\end{array}$ & $\begin{array}{l}4.66 \\
(7)\end{array}$ & $\begin{array}{c}3.47 \\
(5)\end{array}$ & $\begin{array}{c}53.26 \\
(16)\end{array}$ & $\begin{array}{c}1.08 \\
(1)\end{array}$ & $\begin{array}{l}7.92 \\
(12)\end{array}$ & $\begin{array}{l}7.60 \\
(11)\end{array}$ & $\begin{array}{l}6.08 \\
(10)\end{array}$ & $\begin{array}{l}4.12 \\
(5)\end{array}$ & $\begin{array}{c}5.43 \\
(8)\end{array}$ & $\begin{array}{l}5.43 \\
(8)\end{array}$ & $\begin{array}{c}18.12 \\
(14)\end{array}$ & $\begin{array}{c}2.82 \\
(3)\end{array}$ & $\begin{array}{c}13.31 \\
(13)\end{array}$ \\
\hline Diabetes & $\begin{array}{c}25.52 \\
(8)\end{array}$ & $\begin{array}{c}29.16 \\
(12)\end{array}$ & $\begin{array}{c}39.16 \\
(16)\end{array}$ & $\begin{array}{c}34.05 \\
(14)\end{array}$ & $\begin{array}{c}26.87 \\
(10)\end{array}$ & $\begin{array}{c}27.08 \\
(11)\end{array}$ & $\begin{array}{c}25.52 \\
(8)\end{array}$ & $\begin{array}{c}29.31 \\
(13)\end{array}$ & $\begin{array}{c}34.37 \\
(15)\end{array}$ & $\begin{array}{c}21.77 \\
(4)\end{array}$ & $\begin{array}{c}20.88 \\
(2)\end{array}$ & $\begin{array}{c}22.39 \\
(6)\end{array}$ & $\begin{array}{c}21.88 \\
(5)\end{array}$ & $\begin{array}{c}24.60 \\
(7)\end{array}$ & $\begin{array}{c}16.35 \\
(1)\end{array}$ & $\begin{array}{c}21.22 \\
(3)\end{array}$ \\
\hline E. coli & $\begin{array}{c}17.07 \\
(10)\end{array}$ & $\begin{array}{c}13.53 \\
(7)\end{array}$ & $\begin{array}{c}24.38 \\
(15)\end{array}$ & $\begin{array}{c}18.29 \\
(13)\end{array}$ & $\begin{array}{c}15.36 \\
(9)\end{array}$ & $\begin{array}{c}31.70 \\
(16)\end{array}$ & $\begin{array}{c}20.73 \\
(14)\end{array}$ & $\begin{array}{c}17.07 \\
(10)\end{array}$ & $\begin{array}{c}17.07 \\
(10)\end{array}$ & $\begin{array}{c}13.90 \\
(8)\end{array}$ & $\begin{array}{c}7.31 \\
(4)\end{array}$ & $\begin{array}{c}13.41 \\
(6)\end{array}$ & $\begin{array}{c}8.54 \\
(5)\end{array}$ & $\begin{array}{c}7.11 \\
(3)\end{array}$ & $\begin{array}{c}3.11 \\
(1)\end{array}$ & $\begin{array}{l}5.60 \\
(2)\end{array}$ \\
\hline Glass & $\begin{array}{c}29.62 \\
(7)\end{array}$ & $\begin{array}{c}28.51 \\
(6)\end{array}$ & $\begin{array}{c}44.44 \\
(15)\end{array}$ & $\begin{array}{c}17.58 \\
(1)\end{array}$ & $\begin{array}{c}25.36 \\
(3)\end{array}$ & $\begin{array}{c}53.70 \\
(16)\end{array}$ & $\begin{array}{c}24.07 \\
(2)\end{array}$ & $\begin{array}{c}31.66 \\
(9)\end{array}$ & $\begin{array}{c}41.11 \\
(13)\end{array}$ & $\begin{array}{c}38.67 \\
(12)\end{array}$ & $\begin{array}{c}31.89 \\
(10)\end{array}$ & $\begin{array}{c}41.50 \\
(14)\end{array}$ & $\begin{array}{c}37.73 \\
(11)\end{array}$ & $\begin{array}{c}30.79 \\
(8)\end{array}$ & $\begin{array}{c}26.57 \\
(5)\end{array}$ & $\begin{array}{c}26.13 \\
(4)\end{array}$ \\
\hline Horse & $\begin{array}{c}30.76 \\
(3)\end{array}$ & $\begin{array}{c}32.19 \\
(6)\end{array}$ & $\begin{array}{c}38.46 \\
(13)\end{array}$ & $\begin{array}{c}35.71 \\
(11)\end{array}$ & $\begin{array}{c}30.32 \\
(2)\end{array}$ & $\begin{array}{c}38.46 \\
(13)\end{array}$ & $\begin{array}{c}31.86 \\
(4)\end{array}$ & $\begin{array}{c}31.86 \\
(4)\end{array}$ & $\begin{array}{c}41.75 \\
(16)\end{array}$ & $\begin{array}{c}35.16 \\
(10)\end{array}$ & $\begin{array}{c}33.15 \\
(8)\end{array}$ & $\begin{array}{c}38.26 \\
(12)\end{array}$ & $\begin{array}{c}32.97 \\
(7)\end{array}$ & $\begin{array}{c}38.71 \\
(15)\end{array}$ & $\begin{array}{c}29.90 \\
(1)\end{array}$ & $\begin{array}{c}33.44 \\
(9)\end{array}$ \\
\hline Iris & $\begin{array}{l}2.63 \\
(11)\end{array}$ & $\begin{array}{c}0 \\
(1)\end{array}$ & $\begin{array}{l}9.99 \\
(16)\end{array}$ & $\begin{array}{c}0.52 \\
(9)\end{array}$ & $\begin{array}{c}0.26 \\
(8)\end{array}$ & $\begin{array}{l}2.63 \\
(11)\end{array}$ & $\begin{array}{l}2.63 \\
(11)\end{array}$ & $\begin{array}{c}0.52 \\
(9)\end{array}$ & $\begin{array}{c}0 \\
(1)\end{array}$ & $\begin{array}{l}5.26 \\
(15)\end{array}$ & $\begin{array}{l}2.89 \\
(14)\end{array}$ & $\begin{array}{c}0 \\
(1)\end{array}$ & $\begin{array}{c}0 \\
(1)\end{array}$ & $\begin{array}{c}0 \\
(1)\end{array}$ & $\begin{array}{c}0 \\
(1)\end{array}$ & $\begin{array}{c}0 \\
(1)\end{array}$ \\
\hline Thyroid & $\begin{array}{l}6.66 \\
(10)\end{array}$ & $\begin{array}{c}1.85 \\
(4)\end{array}$ & $\begin{array}{c}5.55 \\
(9)\end{array}$ & $\begin{array}{c}13.32 \\
(15)\end{array}$ & $\begin{array}{c}14.62 \\
(16)\end{array}$ & $\begin{array}{l}7.40 \\
(11)\end{array}$ & $\begin{array}{c}11.11 \\
(13)\end{array}$ & $\begin{array}{l}8.51 \\
(12)\end{array}$ & $\begin{array}{c}11.11 \\
(13)\end{array}$ & $\begin{array}{c}3.88 \\
(8)\end{array}$ & $\begin{array}{c}3.14 \\
(6)\end{array}$ & $\begin{array}{c}3.77 \\
(7)\end{array}$ & $\begin{array}{c}0 \\
(1)\end{array}$ & $\begin{array}{c}3.01 \\
(5)\end{array}$ & $\begin{array}{c}0.21 \\
(3)\end{array}$ & $\begin{array}{c}0 \\
(1)\end{array}$ \\
\hline Wine & $\begin{array}{c}0 \\
(1)\end{array}$ & $\begin{array}{c}1.33 \\
(6)\end{array}$ & $\begin{array}{l}2.88 \\
(11)\end{array}$ & $\begin{array}{l}3.99 \\
(13)\end{array}$ & $\begin{array}{l}2.66 \\
(10)\end{array}$ & $\begin{array}{c}17.77 \\
(16)\end{array}$ & $\begin{array}{c}2.22 \\
(8)\end{array}$ & $\begin{array}{l}5.10 \\
(14)\end{array}$ & $\begin{array}{l}5.77 \\
(15)\end{array}$ & $\begin{array}{l}2.88 \\
(11)\end{array}$ & $\begin{array}{c}1.58 \\
(7)\end{array}$ & $\begin{array}{c}0 \\
(1)\end{array}$ & $\begin{array}{c}0 \\
(1)\end{array}$ & $\begin{array}{c}2.64 \\
(9)\end{array}$ & $\begin{array}{c}0 \\
(1)\end{array}$ & $\begin{array}{c}0 \\
(1)\end{array}$ \\
\hline
\end{tabular}


Table 15. Average classification error percentages in all data sets for each of the 15 different classifiers along with the new relative obtained ranks.

\begin{tabular}{|c|c|c|c|c|c|c|c|c|c|c|c|c|c|c|c|c|}
\hline \multirow[b]{2}{*}{ Dataset } & \multicolumn{16}{|c|}{ Classifier } \\
\hline & $\begin{array}{c}\text { Bayes } \\
\text { net }\end{array}$ & $\begin{array}{l}\text { MLP } \\
\text { ANN }\end{array}$ & $\mathrm{RBF}$ & $\begin{array}{c}\text { K } \\
\text { Star }\end{array}$ & Bagging & $\begin{array}{l}\text { Multi } \\
\text { boost }\end{array}$ & $\begin{array}{l}\text { NB } \\
\text { tree }\end{array}$ & Ridor & VFI & PSO & GSA & $\mathrm{ABC}$ & FA & ICA & GGSA & EICA \\
\hline Average & 12.73 & 11.75 & 25.40 & 13.71 & 12.73 & 23.30 & 14.04 & 14.75 & 18.92 & 13.80 & 11.21 & 13.02 & 11.20 & 13.32 & 8.52 & 10.50 \\
\hline Rank & 6 & 5 & 16 & 10 & 6 & 15 & 12 & 13 & 14 & 11 & 4 & 8 & 3 & 9 & 1 & 2 \\
\hline
\end{tabular}

Table 16. The sum of rankings of Table 12 on each column.

\begin{tabular}{|c|c|c|c|c|c|c|c|c|c|c|c|c|c|c|c|c|}
\hline \multirow[b]{2}{*}{ Dataset } & \multicolumn{16}{|c|}{ Classifier } \\
\hline & $\begin{array}{c}\text { Bayes } \\
\text { net }\end{array}$ & $\begin{array}{l}\text { MLP } \\
\text { ANN }\end{array}$ & RBF & $\begin{array}{c}K \\
\text { Star }\end{array}$ & Bagging & $\begin{array}{l}\text { Multi } \\
\text { boost }\end{array}$ & $\begin{array}{l}\text { NB } \\
\text { tree }\end{array}$ & Ridor & VFI & PSO & GSA & $\mathrm{ABC}$ & FA & ICA & GGSA & EICA \\
\hline Average & 80 & 78 & 154 & 114 & 92 & 152 & 113 & 126 & 151 & 114 & 80 & 78 & 52 & 95 & 25 & 50 \\
\hline Rank & 6 & 4 & 16 & 11 & 8 & 15 & 10 & 13 & 14 & 11 & 6 & 4 & 3 & 9 & 1 & 2 \\
\hline
\end{tabular}

the second one is based on the sum of ranking of the techniques. In Table 15, the first and second rows are the average of each column of Table 14 and the general ranking achieved by techniques based on the first row, respectively. Table 16 is also based on the sum of ranking of all the techniques in Table 14. It is obvious that the lower rank is better. At a glance, the proposed EICA algorithm stands alone in the second rank after GGSA algorithm compared to other algorithms.

The computational results demonstrate that the proposed classifier is accurate and reliable. In other words, EICA obtains a good CEP value and ranks the first in most of the datasets, while the other techniques that are specifically tailored for classification do not achieve any first-ranking (i.e., RBF, MultiBoost, Ridor, and PSO).

\subsection{Discussion}

In this paper, a prototype-based classification using extended imperialist has been proposed. According to the results of Subsection 6.2, EICA algorithm significantly outperforms the traditional ICA. There are two major differences that might contribute to the better performance of the EICA.

The first reason is that EICA uses an efficient assimilation function to increase the searching around the imperialist. This function not only uses different random values, but also utilizes the orthogonal colonyimperialistic contacting line instead of $\theta$ for deviating the colony.

The second reason is the new revolution strategy in the EICA. In this strategy, in order to balance the trade-off between the exploration and exploitation of the EICA, a proper method is considered to control the usage rate of search parameter.

The proposed EICA algorithm, as a generalpurpose technique, obtains good result, and shows that it can be successfully applied as classifier to classification problems. However, we cannot guarantee that our algorithm copes with all limitations.

For example, EICA behavior, in terms of problem size $(H)$, in which $H=O \times S$, and the number of cluster $(K)$ in different datasets are compared to those of other optimization algorithms. Consequently, it seems that the results of EICA are better for the datasets with intermediate $H$ values, and its accuracy tends to decrease with an increasing number of cluster.

An important research issue is the investigation of the reasons for the above-mentioned behavior. A sensible hypothesis is that this depends on the number of prototypes used. Using one prototype per class is simple. But, when the class distributions differ from each other, either in the number of patterns, the density of the patterns, or the shape of the classes, the optimal number of prototypes may be different from each other.

Another hypothesis is that this behavior depends on the length of a solution. The length of a solution is proportional to the number of clusters. A large number of clusters complicate the search space, and more execution iteration is required to obtain a satisfying solution.

In the first case, automatically learn the number of prototypes needed to represent every class if needed; allocate different number of prototypes for different classes with different distributions or shapes. In the second case, more iteration might be necessary to obtain a satisfying solution. Hence, the first research issue for our future work should be to find possible ways to get rid of this limitation.

\section{Conclusion}

Classification is one of the robust methods applied by many researchers in several scientific fields. Recently, many of optimization algorithms have been 
proposed based on the simulation of the natural processes in some of the prototype based techniques. In this research, the Extended Imperialist Competitive Algorithm (EICA) is employed to solve the problem of classification. The efficiency of the proposed EICA-based classifier is compared with the best-developed optimization-based classifiers including ABC, FA, PSO, GSA, GGSA, original ICA, and nine well-known classification techniques from the literature; the following results were obtained.

The quality of the result obtained by our proposed algorithm based on the fitness function $f_{1}, f_{2}$, and $f_{3}$ is much better than the other optimization algorithms in some of datasets.

EICA algorithm significantly outperforms traditional ICA in terms of the best and average CEP found using $f_{1}, f_{2}$, and $f_{3}$ after more than 20 different simulations in each dataset.

EICA algorithm has better performance than that of the classification techniques in about $40 \%$ of dataset.

Some relationships between problem size, number of cluster in different datasets, and optimization algorithm performance have been hypothesized in comments on experimental results. However, it seems that the accuracy of the optimization algorithms tends to decrease with increasing values' problem size and number of clusters. The first research issue for our future work should be to find possible ways to get rid of this limitation. Another suggestion for future research is to compare EICA with some relevant papers that modified revolution strategy.

\section{References}

1. Michie, D., Spiegelhalter, D.J. and Taylor, C.C., Machine Learning, Neural and Statistical Classification, Englewood, NJ: Prentice Hall (1994).

2. Mitchell, T., Machine Learning, McGraw-Hill, ISBN 0070428077 (1997).

3. Duda, R.O., Hart, P.E. and Stork, D.G., Pattern Classification, Second Ed., Wiley, New York (2001).

4. Hastie, T., Tibshirani, R. and Friedman, J., The Elements of Statistical Learning: Data Mining, Inference, and Prediction, Springer (2001).

5. Phyu, T. "Survey of classification techniques in data mining", Proceedings of the International Multi Conference of Engineers and Computer Scientists, I, IMECS 2009, Hong Kong, March, pp. 18-20 (2009).

6. Gun, G., Ma, C. and Wu, J. "Data clustering: Theory, algorithms, and applications", Asa-Siam Series on Statistics and Applied Probability (2007).

7. Jain, A. "Data clustering: 50 years beyond k-means", Pattern Recognition, Lett, 31, pp. 651-666 (2010).

8. Jain, A.K., Murty, M.N. and Flynn, P.J. "Data clustering: a review", ACM Comput. Surv., 3, pp. 264323 (1999).
9. Wu, J.Y. "MIMO CMAC neural network classifier for solving classification problems", Applied Soft Computing, 2, pp. 2326-2333 (2011).

10. Lu, C.J. and Wu, J.Y. "An efficient CMAC neural network for stock index forecasting", Expert Systems with Applications, 38, pp. 15194-15201 (2011).

11. Justino, E.J.R., Bortolozzi, F. and Sabourin, R. "A comparison of SVM and HMM classifiers in the off-line signature verification", Pattern Recognition Letters, 26, pp. $1377-1385$ (2005).

12. Mousavi, S.M., Tavakkoli-Moghaddam, R., Vahdani, B., Hashemi, H. and Sanjari, M.J. "New support vector model-based imperialist competitive algorithm for time estimation in new product development projects", Robotics and Computer-Integrated Manufacturing, 29, pp. 157-168 (2013).

13. Saffavian, S.R. and Landgrebe, D. "A survey of decision tree classifier methodology", IEEE Transactions on Systems, Man, and Cybernetics, 3, pp. 660-674 (1991).

14. Kurzynski, M.W. "The optimal strategy of a tree classifier", Pattern Recognition, 16, pp. 81-87 (1983).

15. Aha, D. and Kibler, D. "Instance-based learning algorithms", Machine Learning, 6, pp. 37-66 (1991).

16. Gagliardi, F. "Instance-based classifiers applied to medical databases: Diagnosis and knowledge extraction", Artificial Intelligence in Medicine, 52, pp. 123139 (2011).

17. Shen, F. and Hasegawa, O. "A fast nearest neighbor classifier based on self-organizing incremental neural network", Neural Networks, 10, 1537-1547 (2008).

18. Chou, C.H., Lin, C.C., Liu, Y.H. and Chang, F. "A prototype classification method and its use in a hybrid solution for multiclass pattern recognition", Pattern Recognition, 39, pp. 624-634 (2006).

19. Vapnik, V.N., The Nature of Statistical Learning Theory, Springer, Berlin, (1995).

20. Borgelt, C. "Prototype-based classification and clustering", Ph.D. Thesis, Ottovon-Guericke University Magdeburg (November 2005).

21. Nieddu, L. and Patrizi, G. "Formal methods in pattern recognition: A review", European Journal of Operational Research, 120, pp. 459-495 (2000).

22. Gagliardi, F. "Instance-based classifiers to discover the gradient of typicality in data", Artificial Intelligence Around Man and Beyond, 6934, pp. 457-462 (2011).

23. Song, H.H. and Lee, S.W. "LVQ combined with simulated annealing for optimal design of large-set reference models", Neural Networks, 2, pp. 329-336 (1996).

24. Cover, T.M. and Hart, P.E. "Nearest neighbor pattern classification", IEEE Transactions, Inform. Theory, 13, pp. 21-27 (1967). 
25. Veenman, C.J. and Reinders, M.J.T. "The nearest subclass classifier: A compromise between the nearest mean and nearest neighbor classifier", IEEE Transactions on Pattern Analysis and Machine Intelligence, $\mathbf{9}$, pp. 1417-1429 (2005).

26. Bezdek, J.C. and Kuncheva, L.I. "Nearest prototype classifier design: An experimental study", International Journal of Intelligent Systems, 16, pp. 14451473 (2001).

27. Wilson, D.R. and Martinez, T.R. "Reduction techniques for instance-based learning algorithms", $M a$ chine Learning, 38, pp. 257-286 (2000).

28. Bahrololoum, A., Nezamabadi-pour, H., Bahrololoum, H. and Saeed, M. "A prototype classifier based on gravitational search algorithm", Applied Soft Computing, 12, pp. 819-825 (2012).

29. De Falco, I., Della Cioppa, A. and Tarantino, E. "Facing classification problems with particle swarm optimization", Applied Soft Computing, 3, pp. 652-658 (2007).

30. Karaboga, D. and Ozturk, C. "A novel clustering approach: Artificial bee colony (ABC) algorithm", Applied Soft Computing, 11, pp. 652-657 (2011).

31. Senthilnath, J., Omkar, S.N. and Mani, V. "Clustering using firefly algorithm: Performance study", Swarm and Evolutionary Computation, 1, pp. 164-171 (2011).

32. Dowlatshahi, M.B. and Nezamabadi-pour, H. "GGSA: A Grouping Gravitational Search Algorithm for data clustering", Engineering Applications of Artificial Intelligence, 36, pp. 114-121 (2014).

33. Atashpaz-Gargari, E., and Lucas, C. "Imperialist competitive algorithm: An algorithm for optimization inspired by imperialist competitive", IEEE Congress on Evolutionary Computation, Singapore (2007).

34. Atashpaz-Gargari, E., Hashemzadeh, F., Rajabioun, R. and Lucas, C. "Colonial competitive algorithm: A novel approach for PID controller design in MIMO distillation column process", Int. J. Intell. Comput. Cybernet, 1, pp. 337-55 (2008).

35. Alaghebandha, M. and Hajipour, V. "A soft computing-based approach to optimise queuing inventory control problem", International Journal of Systems Science, 46, pp. 1113-1130 (2013).

36. Niknam, T., TaherianFard, E., Pourjafarian, N. and Rousta, A. "An efficient hybrid algorithm based on modified imperialist competitive algorithm and Kmeans for data clustering", Engineering Applications of Artificial Intelligence, 24, pp. 306-317 (2011).

37. Behnamian, J. and Zandieh, M. "A discrete colonial competitive algorithm for hybrid flow shop scheduling to minimize earliness and quadratic tardiness penalties", Expert Systems with Applications, 12, pp. 1449014498 (2011).
38. Afonso, L.D., Mariani, V.C. and Coelho, L.D.S. "Modified imperialist competitive algorithm based on attraction and repulsion concepts for reliability-redundancy optimization", Expert Systems with Applications, 40, pp. 3794-3802 (2013).

39. Rajabioun, R., Atashpaz-Gargari, E. and Lucas, C. "Colonial competitive algorithm as a tool for Nash equilibrium point achievement", Computational Science and Its Applications, 5073, pp. 680-695 (2008).

40. Naimi Sadigh, A., Mozafari, M. and Karimi, B. "Manufacturer-retailer supply chain coordination: A bi-level programming approach", Advances in Engineering Software, 45, 144-152 (2012).

41. Rahimi, A., Karimi, H. and Afshar-Nadjafi, B. "Using meta-heuristics for project scheduling under mode identity constraints", Applied Soft Computing, 13, pp. 2124-2135 (2013).

42. Hosseini, S., Khaled, A. and Vadlamani, S. "Hybrid imperialist competitive algorithm, variable neighborhood search, and simulated annealing for dynamic facility layout problem", Neural Computing and Applications, 7, pp. 1871-1885 (2014).

43. Hosseini, S., Khaled, A.A. and Jin, M. "Solving Euclidean minimal spanning tree problem using a new meta-heuristic approach: Imperislist competitive algorithm (ICA)", IEEE International Conference on Industrial Engineering and Engineering Management, Hong Kong, pp. 10-13 (2012).

44. Khaled, A.A. and Hosseini, S. "Fuzzy adaptive imperialist competitive algorithm for global optimization", Neural Computing and Applications, 26(4), pp. 813825 (2015).

45. Hosseini, S. and Khaled, A. "A survey on the imperialist competitive algorithm metaheuristic: Implementation in engineering domain and directions for future research", Applied Soft Computing, 24, pp. 1078-1094 (2014).

46. Zhang, Y., Wang, Y. and Peng, C. "Improved imperialist competitive algorithm for constrained optimization", In Proceedings of International Forum on Computer Science-Technology and Applications, Chongqing, China, pp. 204-207 (2009).

47. Razzaghpour, M. and Rusu, A. "Analog circuit optimization via a modified imperialist competitive algorithm", IEEE International Symposium on Circuits and Systems, pp. 2273-2276 (2011).

48. Duan, H.B., Xu, C.F., Liu, S.Q. and Shao, S. "Template matching using chaotic imperialist competitive algorithm", Pattern Recognition Lett, 13, pp. 18681875 (2010).

49. Kaveh, A. and Talatahari, S. "Optimum design of skeletal structures using imperialist competitive algorithm", Computers and Structures, 88, pp. 1220-1229 (2010). 
50. Blake, C.L. and Merz, C.J. "University of California at Irvine repository of machine learning databases", http://www.ics.uci.edu/ mlearn/MLRepository. Html (1998).

51. Nazari-Shirkouhi, S., Eivazy, H., Ghodsi, R., Rezaie, K. and Atashpaz-Gargari, E. "Solving the integrated product mix-outsourcing problem using the Imperialist competitive algorithm", Expert Systems with Applications, 37, pp. 7615-7626 (2010).

52. Ben-Arieh, D. and Gullipalli, D.K. "Data envelopment analysis of clinics with sparse data: Fuzzy clustering approach", Computers \& Industrial Engineering, 63, pp. 13-21 (2012).

53. Hulse, J.V. and Khoshgoftaar, T.M. "Incomplete-case nearest neighbor imputation in software measurement data", Information Sciences, 259, pp. 410-596 (2014).

54. Eirola, E., Lendasse, A., Vandewalle, V. and Biernacki, V. "Mixture of Gaussians for distance estimation with missing data", Neurocomputing, 131, pp. 32-42 (2014).

55. Ensen, F., An Introduction to Bayesian Networks, UCL Press/Springer-Verlag (1996).

56. Rumelhart, D.E., Hinton, G.E. and Williams, R.J. "Learning representation by back-propagation errors", Nature, 323, pp. 533-536 (1986).

57. Hassoun, M.H., Fundamentals of Artificial Neural Networks, The MIT Press, Cambridge, MA (1995).

58. Cleary, J.G. and Trigg, L.E. " $K^{*}$ : An instancebased learner using an entropic distance measure", In Proceedings of the 12th International Conference on Machine Learning, pp. 108-114 (1995).

59. Breiman, L. "Bagging predictors", Machine Learn, 2, pp. 123-140 (1996).

60. Webb, G.I. "Multiboosting: A technique for combining boosting and wagging", Machine Learn, 2, pp. 159-196 (2000).

61. Kohavi, R. "Scaling up the accuracy of naive-bayes classifiers: a decision tree hybrid", Proceedings of the Second International Conference on Knowledge Discovery and Data Mining, AAAI Press, pp. 202-207 (1996).

62. Compton, P. and Jansen, R. "Knowledge in context: a strategy for expert system maintenance", Proceedings of Artificial Intelligence, 406, LNAI, Berlin, SpringerVerlag, Adelaide, Australia, pp. 292-306 (1988).

63. Demiroz, G. and Guvenir, A. "Classification by voting feature intervals", In Proceedings of the Seventh European Conference on Machine Learning, pp. 85-92 (1997).

64. Garcia, S., Fernandez, A., Luengo, J. and Herrera, F. "Advanced nonparametric tests for multiple comparisons in the design of experiments in computational intelligence and data mining: experimental analysis of power", Information Sciences, 180(10), pp. 2044-2064 (2010).
65. Friedman, M. "A comparison of alternative tests of significance for the problem of m rankings", Annals of Mathematical Statistics, 11, pp. 86-92 (1940).

66. Iman, R. and Davenport, J. "Approximations of the critical region of the Friedman statistic", Communications in Statistics, 9, pp. 571-595 (1980).

\section{Biographies}

Mohammad Hossein Fazel Zarandi is a Full Professor in the Department of Industrial Engineering at Amirkabir University of Technology, Tehran, Iran, and a member of the Knowledge-Intelligent Systems Laboratory at Department of Mechanical and Industrial Engineering of University of Toronto, Canada. His main research interests focus on: intelligent information systems, soft computing and computational intelligence, Type- 1 and Type-2 fuzzy sets and systems, artificial intelligence and expert systems, multi-agent systems, networks, big data modeling, data mining, fuzzy rough sets, meta-heuristics, and optimization. Professor Fazel Zarandi has authored many books, scientific journal and referred international conference papers, and technical reports in the above areas, most of which are available on the web. He has taught several courses at several universities in Iran and North America on fuzzy systems engineering, decision support systems, management information systems, artificial intelligence and expert systems, systems analysis and design, scheduling, neural networks, simulations, and production planning and control.

Mohammad Teimouri received his BSc in Shomal University on 2009 and MSc degrees in Industrial Engineering from Islamic Azad University, Qazvin Branch in 2013, respectively. His research interests are in data mining, combinatorial optimization, and reliability engineering.

Arash Zaretalab is a $\mathrm{PhD}$ candidate at the Department of Industrial Engineering at Amirkabir University of technology (Tehran polytechnic) in Iran. He received his BSc and MSc degrees in Industrial Engineering from Islamic Azad University, Qazvin Branch (IAU). His research interests are in reliability engineering, combinatorial optimization, multi-objective optimization, computational intelligence, and data mining. Mr. Zaretalab has published a number of papers in journals such as reliability engineering \& safety system, computer operation research, computers \& industrial engineering among others.

Vahid Hajipour is a $\mathrm{PhD}$ candidate in the department of Industrial Engineering at Bu-Ali Sina University in Iran. He is also a Visiting Professor of Industrial and Mechanical Engineering at the Islamic Azad 
University (IAU). He holds both MS and BS degrees in Industrial Engineering from the IAU. Mr. Hajipour is a member of the Iranian National Elite Foundation and Young Researchers Club. He is the recipient of the 2011, 2013, and 2014 Distinguished Young Researcher Award at the IAU. His areas of interest include queuing theory, facility location modelling, supply chain network design, reliability theory, multi-objective optimization, computational intelligence, and fuzzy sets theory. Mr. Hajipour has published one book and more than 38 papers in reputable academic journals and conferences such as Applied Mathematical Modelling, Computer and Industrial Engineering, IEEE Transaction of Engineering Management, Journal of Intelligent Manufacturing, Applied Soft Computing, International Journal of System Science, Measurement, International Journal of Advanced Manufacturing Technology, and among others. 